University of Nebraska - Lincoln

DigitalCommons@University of Nebraska - Lincoln

2005

\title{
Mercury Concentrations in Fishes from Select Water Bodies in Trinity County, California, 2000-2002
}

Jason T. May

U.S. Geological Survey

Roger L. Hothem

U.S. Geological Survey

Charles N. Alpers

U.S. Geological Survey

Follow this and additional works at: https://digitalcommons.unl.edu/usgspubs

Part of the Earth Sciences Commons

May, Jason T.; Hothem, Roger L.; and Alpers, Charles N., "Mercury Concentrations in Fishes from Select Water Bodies in Trinity County, California, 2000-2002" (2005). Publications of the US Geological Survey. 60 .

https://digitalcommons.unl.edu/usgspubs/60

This Article is brought to you for free and open access by the US Geological Survey at DigitalCommons@University of Nebraska - Lincoln. It has been accepted for inclusion in Publications of the US Geological Survey by an authorized administrator of DigitalCommons@University of Nebraska - Lincoln. 
In cooperation with the U.S. Department of Agriculture-Forest Service

\section{Mercury Concentrations in Fishes from Select Water Bodies in Trinity County, California, 2000-2002}

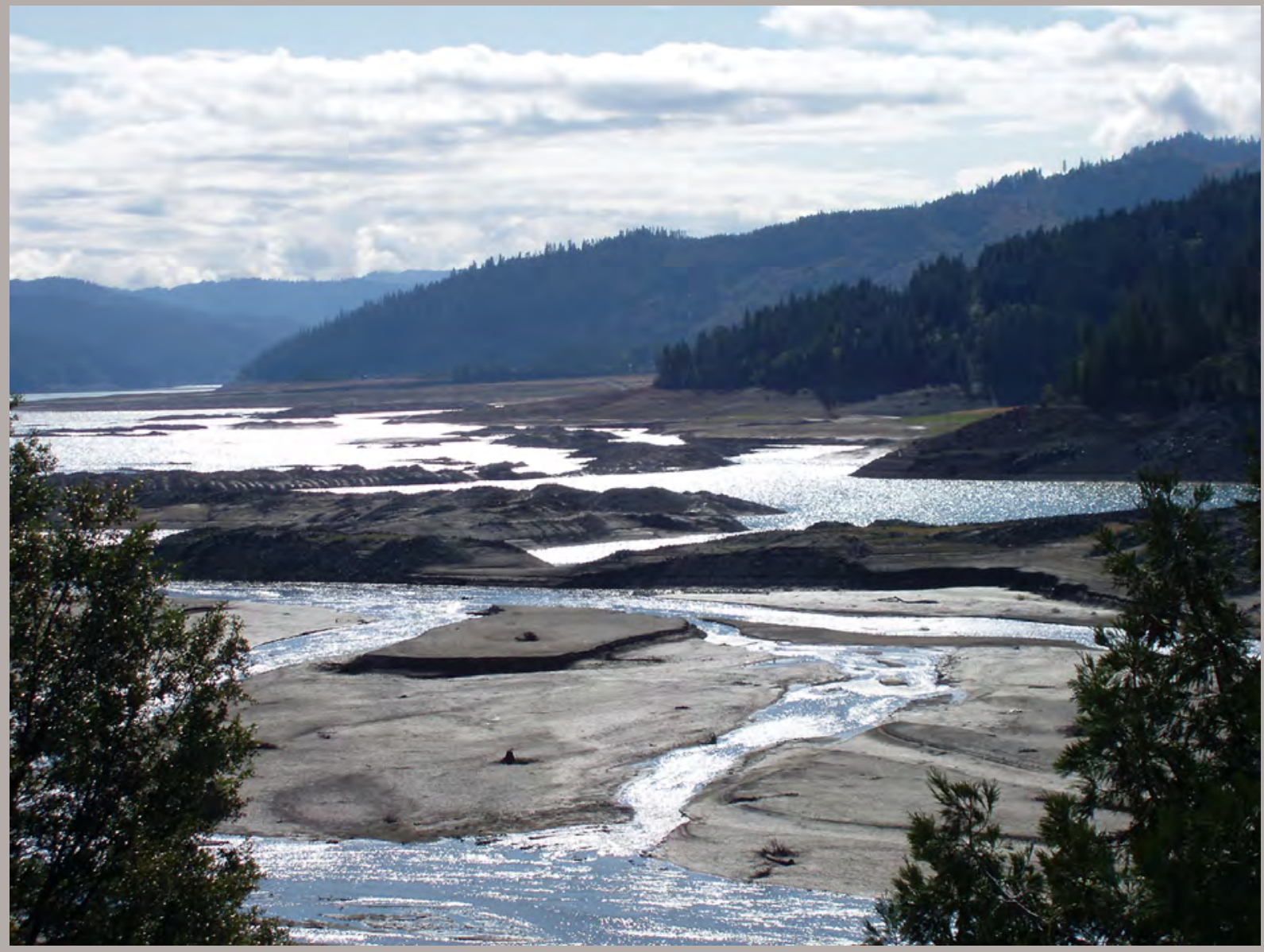

Open-File Report 2005-1321

U.S. Department of the Interior U.S. Geological Survey 
Cover photo: Trinity River arm of Trinity Lake, showing tailings from historical mining of placer gold deposits (Jason May, U.S. Geological Survey). 


\section{Mercury Concentrations in Fishes from Select Water Bodies in Trinity County, California, 2000-2002}

By Jason T. May' ${ }^{1}$ Roger L. Hothem², and Charles N. Alpers',

U.S. Geological Survey Open-File Report 2005-1321

${ }^{1}$ U.S. Geological Survey, California Water Science Center, 6000 J Street, Placer Hall, Sacramento, CA 95819-6129

${ }^{2}$ U.S. Geological Survey, Western Ecological Research Center, Dixon Field Station, 6924 Tremont Road, Dixon, CA 95620

Sacramento, California 


\title{
U.S. Department of the Interior \\ Gale A. Norton, Secretary
}

\author{
U.S. Geological Survey \\ P. Patrick Leahy, Acting Director
}

U.S. Geological Survey, Reston, Virginia: 2005

For Sale by U.S. Geological Survey, Information Services

Box 25286, Denver Federal Center

Denver, CO 80225-0286

For more information about the USGS and its products:

Telephone: 1-888-ASK-USGS

World Wide Web: http://www.usgs.gov

Any use of trade, product, or firm names in this publication is for descriptive purposes only and does not imply endosement by the U.S. Government.

Although this report is in the public domain, permission must be secured from the individual copyright owners to reproduce any copyrighted materials contained within this report.

Suggested citation: May, J.T., Hothem, R.L, and Alpers, C.N., 2005, Mercury Concentrations in Fishes from Select Water Bodies in Trinity County, California, 2000-2002: U.S. Geological Survey Open-File Report 2005-1321, 14 p. 


\section{Contents}

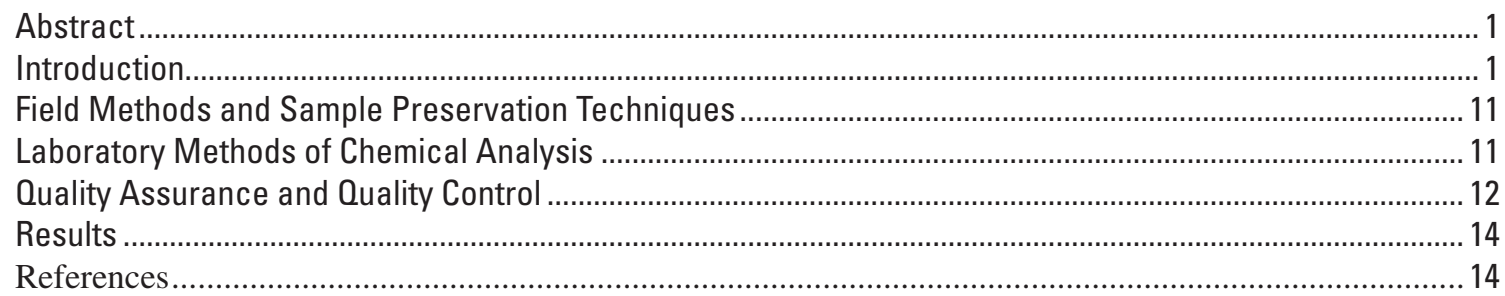

\section{Figure}

1. Map showing fish sampling locations within the Trinity River watershed,

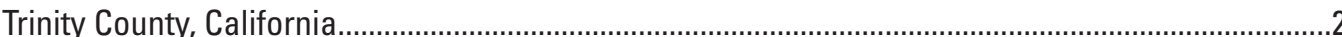

\section{Tables}

1. Fish sampling sites and data-collection years, Trinity County, California, 2000-2002....................................

2.Data for fishes collected in Trinity County, California, 2000-2002 ................................................................

3. Data from replicate analyses of fishes collected in Trinity County, California, 2000-2002...........................12 


\section{Abbrevations and Datum Used}

Horizontal coordinate information is referenced to North American Datum of 1983, (NAD 83)

BLM, Bureau of Land Management

CRV, certified reference value

CVAAS, cold-vapor atomic absorption spectroscopy

LOD, limit of detection

NRCC, National Research Council Canada

OEHHA, Office of Environmental Health Hazard Assessment (California)

QA, quality assurance

$\mathrm{QC}$, quality control

RPD, relative percentage difference

SRM, standard reference material

SWRCB, State Water Resources Control Board (California)

TERL, Trace Element Research Laboratory

USDA-FS, U.S. Department of Agriculture-Forest Service

USEPA, U.S. Environmental Protection Agency

USGS, U.S. Geological Survey

$\mathrm{Hg}$, mercury

$\mathrm{Hg}^{0}$, elemental mercury

$\mathrm{Hg}^{2+}$, mercuric ion

g, gram

$\mathrm{mm}$, millimeter

ppm, part per million

ww, wet weight

$\mu \mathrm{g} / \mathrm{g}$, microgram per gram 


\title{
Mercury Concentrations in Fishes from Select Water Bodies in Trinity County, California, 2000-2002
}

\author{
By Jason T. May, Roger L. Hothem, and Charles N. Alpers
}

\section{Abstract}

Sites of historical gold and mercury mining in the Trinity River watershed continue to release mercury to downstream water bodies. To evaluate the extent of mercury $(\mathrm{Hg})$ contamination in the watershed, the U.S. Geological Survey collected samples of sediment, water, invertebrates, amphibians, and fishes from select water bodies and mine sites in Trinity County, California. This report presents total mercury data for 368 fishes collected during 2000-2002, from 4 locations within Trinity Lake, from 16 stream sites, and from 3 pond sites within the Trinity River watershed. The following species of fish were sampled (scientific name and number of samples in parentheses): brook trout (Salvelinus fontinalis, 13), brown bullhead (Ameiurus nebulosus, 5), green sunfish (Lepomis cyanellus, 13), largemouth bass (Micropterus salmoides, 33), marbled sculpin (Cottus klamathensis, 24), rainbow trout (Oncorhynchus mykiss, 237), smallmouth bass (Micropterus dolomieu, 41), and white catfish (Ameiurus catus, 2). Total mercury in 74 black bass (largemouth and smallmouth bass; Micropterus spp.) samples ranged from 0.046 to 1.225 micrograms per gram (equivalent to parts per million or ppm) wet weight (ww). Mercury concentrations in 26 of the 34 black bass (76 percent) of "legal catch size" ( $\geq 305$ millimeters in length) were $\geq 0.3 \mathrm{ppm}$ (ww), the U.S. Environmental Protection Agency water-quality criterion for the protection of human health Mercury concentrations exceeded $1.0 \mathrm{ppm}(\mathrm{ww})$, the Food and Drug Administration action level for commercial fish in 3 of the 34 black bass ( 9 percent) of legal catch size. In contrast, only 3 of the 237 (about 1 percent) rainbow trout of all sizes sampled from stream, pond, and lake sites had $\mathrm{Hg}$ concentrations $\geq 0.3 \mathrm{ppm}$ (ww). These results indicate that some fish species inhabiting select water bodies of Trinity County contain undesirably high concentrations of mercury in their skinless fillets. In response to data generated by this study and other related investigations, the California Environmental Protection Agency's Office of Environmental Health Hazard Assessment (OEHHA) issued a draft fish-consumption advi- sory report that offered guidelines for human consumption of fish. The final version of the OEHHA fish-consumption advisory was approved by the State of California in July 2005 and is scheduled for publication in October 2005 (http://www.oehha. ca.gov/fish/so_cal/TrinRiverF2.html)

\section{Introduction}

Considerable mining of placer (alluvial) gold deposits, using hydraulic methods that employed mercury to process ore, took place in Trinity County, California, during the latter half of the 19th century and the first part of the 20th century (Clark, 1963; Alpers and others, 2005). In addition, mercury was mined by underground methods in the Altoona Mining district (Swinney, 1950).

During 2000-2002, the U.S. Geological Survey (USGS) conducted an investigation, in cooperation with other federal and state agencies, to assess mercury contamination associated with historical mining in the Trinity River watershed. Agencies that provided funding and in-kind services for the investigation included the U.S. Department of Agriculture-Forest Service (USDA-FS) Shasta-Trinity National Forest, the Bureau of Land Management (BLM), and the California State Water Resources Control Board (SWRCB). During 2000-2002, the USGS collected 368 fishes from 4 locations within Trinity Lake, from 16 stream sites, and from 3 pond sites within the Trinity River watershed (fig. 1; tables 1 and 2). The following species of fish were sampled and analyzed for total mercury (scientific name and number of samples in parentheses): brook trout (Salvelinus fontinalis, 13), brown bullhead (Ameiurus nebulosus, 5), green sunfish (Lepomis cyanellus, 13), largemouth bass (Micropterus salmoides, 33), marbled sculpin (Cottus klamathensis, 24), rainbow trout (Oncorhynchus mykiss, 237), smallmouth bass (Micropterus dolomieu, 41), and white catfish (Ameiurus catus, 2). 


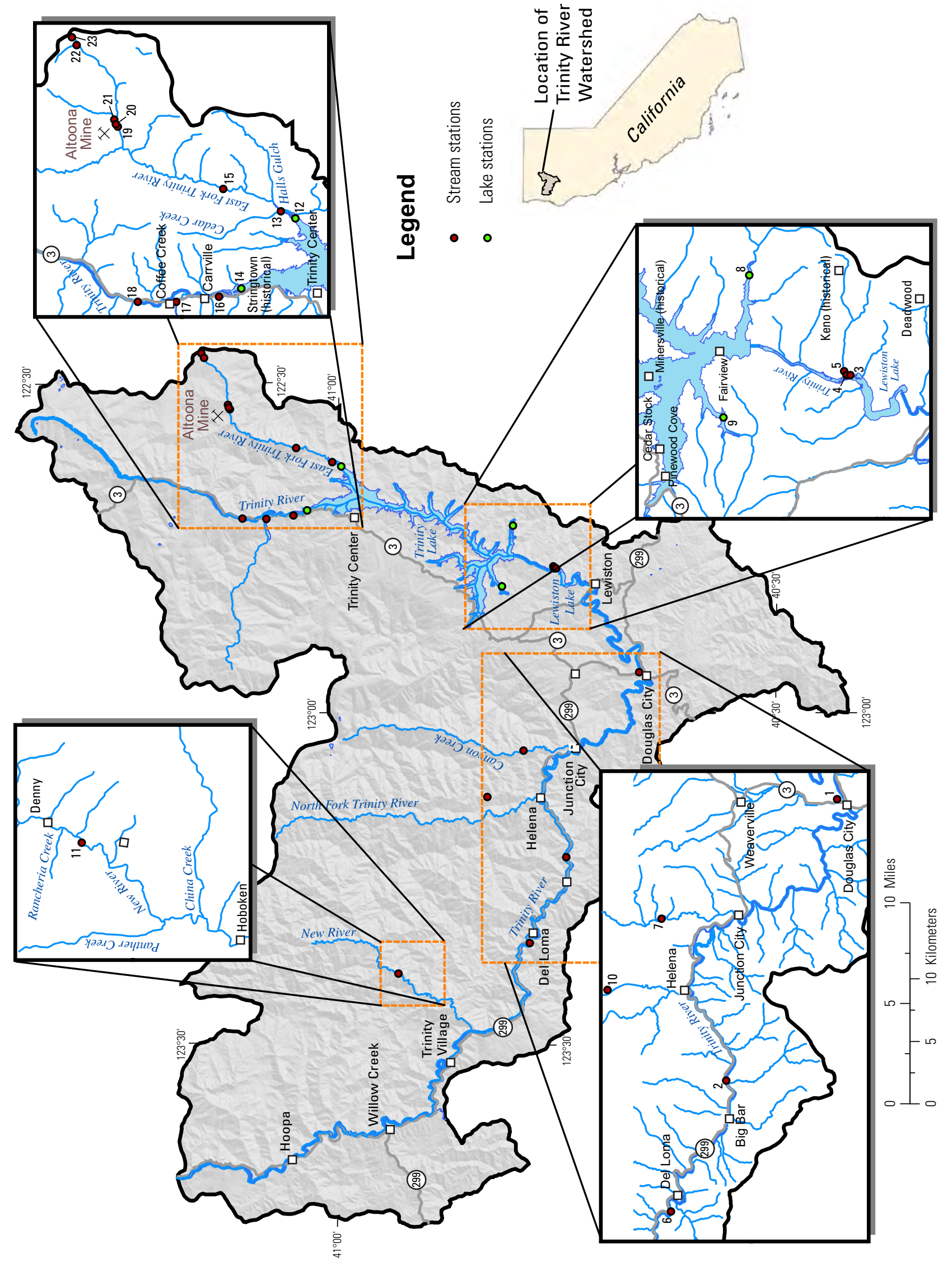

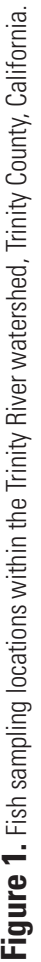


Table 1. Fish sampling sites and data-collection years, Trinity County, California, 2000-2002.

[Hwy, highway; Mt, Mount. USGS, U.S. Geological Survey. Horizontal coordinate information is referenced to the North American Datum of 1983 (NAD83)]

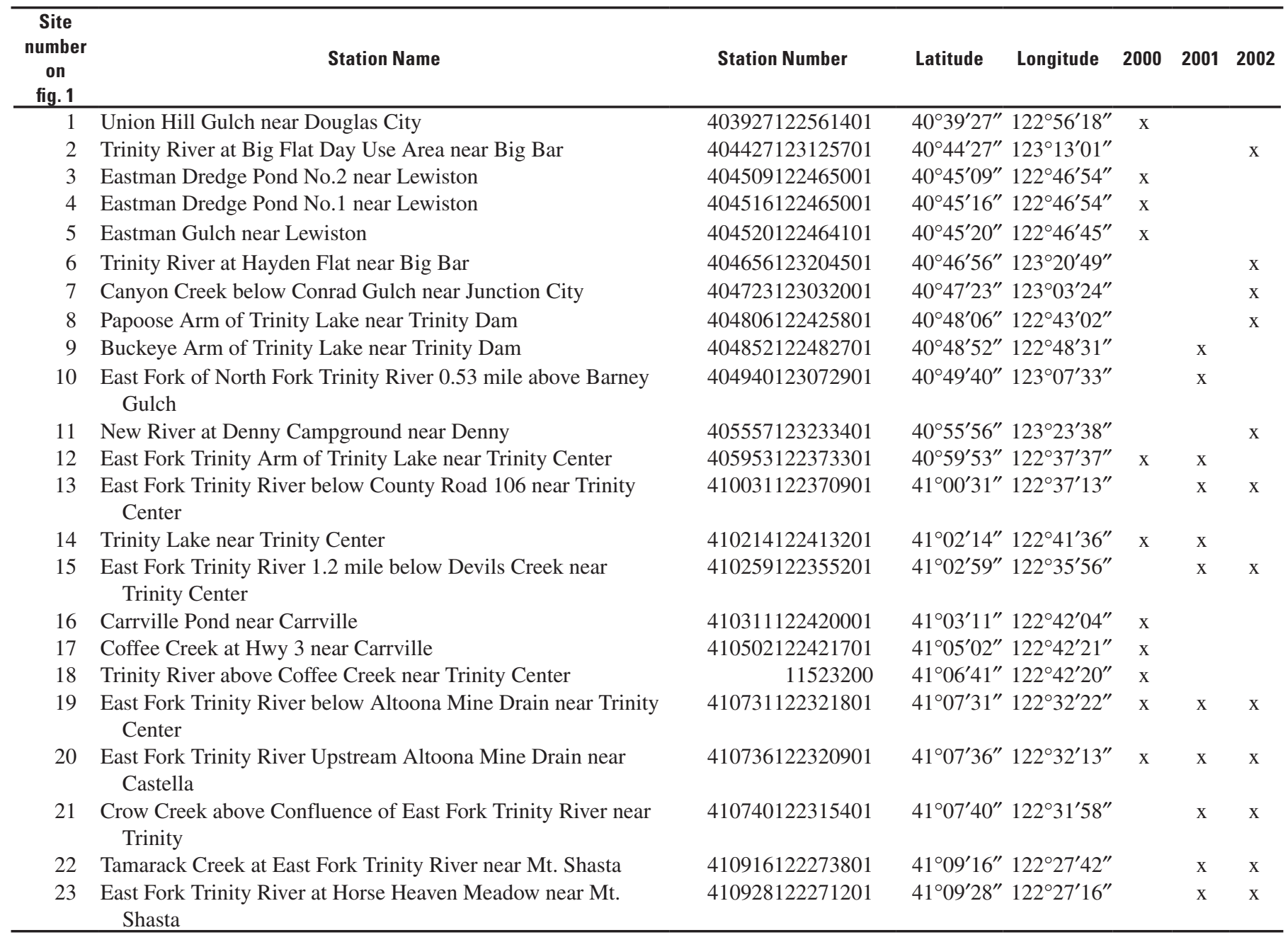

Table 2. Data for fishes collected in Trinity County, California, 2000-2002.

[Fillet samples dissected from left fillet of fish; whole body, whole body fish analyzed with gastrointestinal tract cleaned out. Hwy, highway; Mt, Mount. $\mathrm{Hg}$, mercury; HgT, total mercury. Sex: F, female; M, male; U, unknown. mm, millimeter; g, gram; \%, percent; $\mu \mathrm{g} / \mathrm{g}$, microgram per gram (equivalent to part per million)]

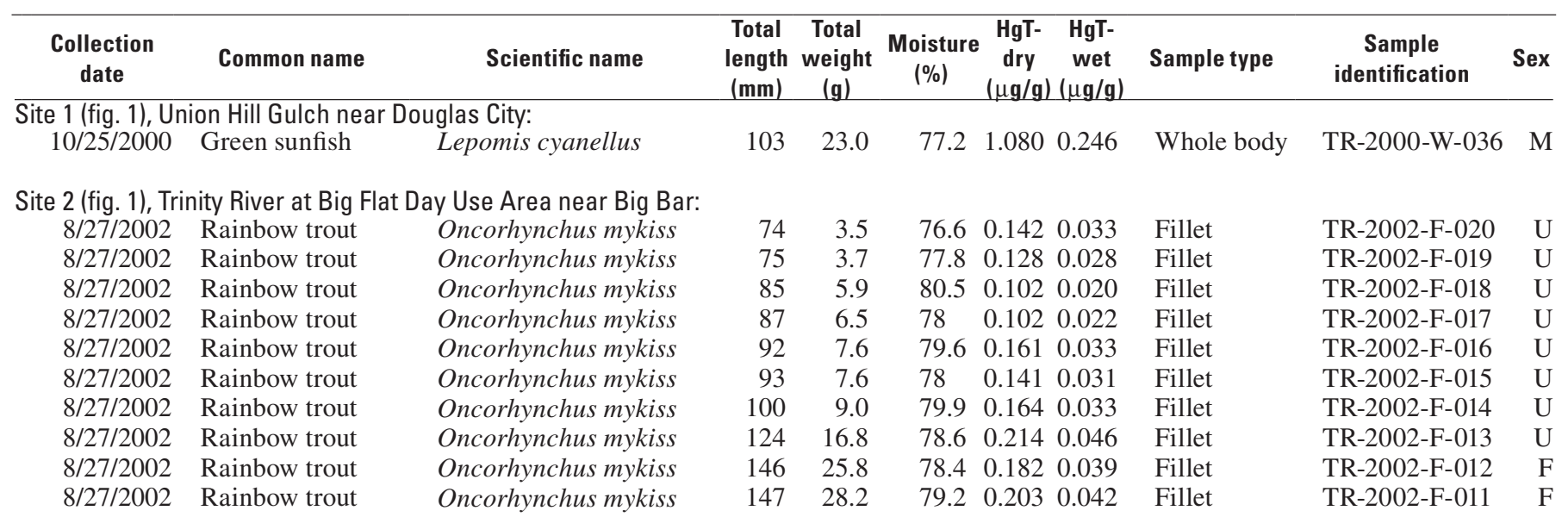


Table 2. Data for fishes collected in Trinity County, California, 2000-2002_Continued.

[Fillet samples dissected from left fillet of fish; whole body, whole body fish analyzed with gastrointestinal tract cleaned out. Hwy, highway; Mt, Mount. $\mathrm{Hg}$, mercury; HgT, total mercury. Sex: F, female; M, male; U, unknown. mm, millimeter; g, gram; \%, percent; $\mu \mathrm{g} / \mathrm{g}$, microgram per gram (equivalent to part per million)]

\begin{tabular}{|c|c|c|c|c|c|c|c|c|c|c|}
\hline $\begin{array}{c}\text { Collection } \\
\text { date }\end{array}$ & Common name & Scientific name & $\begin{array}{l}\text { Total } \\
\text { length } \\
(\mathrm{mm})\end{array}$ & $\begin{array}{c}\text { Total } \\
\text { weight } \\
\text { (g) }\end{array}$ & $\begin{array}{c}\text { Moisture } \\
(\%)\end{array}$ & $\begin{array}{c}\text { HgT- } \\
\text { dry } \\
(\mu \mathrm{g} / \mathrm{g})\end{array}$ & $\begin{array}{c}\text { HgT- } \\
\text { wet } \\
(\mu \mathrm{g} / \mathrm{g})\end{array}$ & Sample type & $\begin{array}{c}\text { Sample } \\
\text { identification }\end{array}$ & Sex \\
\hline \multicolumn{11}{|c|}{ Site 3 (fig. 1), Eastman Dredge Pond No. 2 near Lewiston: } \\
\hline $10 / 23 / 2000$ & Green sunfish & Lepomis cyanellus & 122 & 25.4 & 77.7 & 0.842 & 0.188 & Whole body & TR-2000-W-032 & $\mathrm{F}$ \\
\hline $10 / 23 / 2000$ & Green sunfish & Lepomis cyanellus & 129 & 27.6 & 79.2 & 0.550 & 0.114 & Whole body & TR-2000-W-034 & $\mathrm{U}$ \\
\hline $10 / 23 / 2000$ & Green sunfish & Lepomis cyanellus & 130 & 27.0 & 78.1 & 0.755 & 0.165 & Whole body & TR-2000-W-033 & $\mathrm{F}$ \\
\hline $10 / 23 / 2000$ & Green sunfish & Lepomis cyanellus & 162 & 57.1 & 77.8 & 0.486 & 0.108 & Whole body & TR-2000-W-035 & M \\
\hline
\end{tabular}

Site 3 (fig. 1), Eastman Dredge Pond No. 1 near Lewiston: 9/26/2000 Green sunfish Lepomis cyanellus 9/26/2000 Green sunfish Lepomis cyanellus 9/26/2000 Green sunfish Lepomis cyanellus 10/23/2000 Green sunfish Lepomis cyanellus 10/23/2000 Green sunfish Lepomis cyanellus

$\begin{array}{rrlll}91 & 13.2 & 75 & 0.409 & 0.102 \\ 92 & 15.0 & 76.9 & 0.432 & 0.100 \\ 109 & 21.8 & 76.7 & 0.843 & 0.196 \\ 84 & 8.4 & 74.8 & 0.806 & 0.203 \\ 125 & 29.7 & 77.9 & 0.730 & 0.161\end{array}$

Whole body Whole body Whole body Whole body Whole body

TR-2000-W-039 M TR-2000-W-040 M TR-2000-W-038 F TR-2000-W-041 F TR-2000-W-037 M

Site 5 (fig. 1), Eastman Gulch near Lewiston:

9/26/2000 Rainbow trout Oncorhynchus mykiss 9/26/2000 Rainbow trout Oncorhynchus mykiss 9/26/2000 Rainbow trout Oncorhynchus mykiss 9/26/2000 Rainbow trout Oncorhynchus mykiss 9/26/2000 Rainbow trout Oncorhynchus mykiss

Site 6 (fig. 1), Trinity River at Hayden Flat near Big Bar: 8/27/2002 Rainbow trout Oncorhynchus mykiss 8/27/2002 Rainbow trout Oncorhynchus mykiss 8/27/2002 Rainbow trout Oncorhynchus mykiss 8/27/2002 Rainbow trout Oncorhynchus mykiss 8/27/2002 Rainbow trout Oncorhynchus mykiss 8/27/2002 Rainbow trout Oncorhynchus mykiss $8 / 27 / 2002$ Rainbow trout $8 / 27 / 2002$ Rainbow trout $8 / 27 / 2002$ Rainbow trout 8/27/2002 Rainbow trout Oncorhynchus mykiss Oncorhynchus mykiss Oncorhynchus mykiss Oncorhynchus mykiss

$\begin{array}{lllll}136 & 26.1 & 74.2 & 0.430 & 0.111\end{array}$ $\begin{array}{lllll}140 & 25.7 & 76.3 & 0.420 & 0.100\end{array}$ $\begin{array}{llllll}142 & 30.5 & 74.6 & 0.448 & 0.114\end{array}$ $\begin{array}{lllll}144 & 30.0 & 75.1 & 0.353 & 0.088\end{array}$ $\begin{array}{lllll}150 & 35.5 & 72 & 0.345 & 0.097\end{array}$

$\begin{array}{lllll}91 & 8.1 & 75.5 & 0.132 & 0.032\end{array}$

$\begin{array}{lllll}93 & 8.2 & 76.8 & 0.151 & 0.035\end{array}$

$\begin{array}{lllll}100 & 10.4 & 78.1 & 0.187 & 0.041\end{array}$

$\begin{array}{lllll}102 & 10.5 & 81.5 & 0.350 & 0.065\end{array}$

$\begin{array}{lllll}115 & 15.9 & 79.5 & 0.278 & 0.057\end{array}$

$\begin{array}{lllll}116 & 17.9 & 80 & 0.209 & 0.042\end{array}$

$\begin{array}{llllll}116 & 14.8 & 78.9 & 0.236 & 0.050\end{array}$

$\begin{array}{lllll}137 & 24.7 & 79.1 & 0.250 & 0.052\end{array}$

$\begin{array}{lllll}138 & 25.7 & 76.3 & 0.252 & 0.060\end{array}$

$\begin{array}{lllll}166 & 40.9 & 78.7 & 0.306 & 0.065\end{array}$

Whole body Whole body Whole body Whole body Whole body

Fillet Fillet Fillet Fillet Fillet Fillet Fillet Fillet Fillet Fillet

Site 7 (fig. 1), Canyon Creek below Conrad Gulch near Junction City: $8 / 27 / 2002$ Rainbow trout $8 / 27 / 2002$ Rainbow trout $8 / 27 / 2002$ Rainbow trout $8 / 27 / 2002$ Rainbow trout $8 / 27 / 2002$ Rainbow trout $8 / 27 / 2002$ Rainbow trout $8 / 27 / 2002$ Rainbow trout $8 / 27 / 2002$ Rainbow trout $8 / 27 / 2002$ Rainbow trout 8/27/2002 Rainbow trout Oncorhynchus mykiss Oncorhynchus mykiss Oncorhynchus mykiss Oncorhynchus mykiss Oncorhynchus mykiss Oncorhynchus mykiss Oncorhynchus mykiss Oncorhynchus mykiss Oncorhynchus mykiss Oncorhynchus mykiss

Site 8 (fig. 1), Papoose Arm of Trinity Lake near Trinity Dam: 5/16/2001 Largemouth bass Micropterus salmoides 5/16/2001 Rainbow trout $5 / 16 / 2001$ Rainbow trout $5 / 16 / 2001$ Rainbow trout 5/16/2001 Smallmouth bass 5/16/2001 Smallmouth bass 5/16/2001 Smallmouth bass 5/16/2001 Smallmouth bass 5/16/2001 Smallmouth bass Oncorhynchus mykiss Oncorhynchus mykiss Oncorhynchus mykiss Micropterus dolomieu Micropterus dolomieu Micropterus dolomieu Micropterus dolomieu Micropterus dolomieu $\begin{array}{lllll}113 & 12.5 & 79.5 & 0.176 & 0.036\end{array}$ $126 \quad 18.2$

$\begin{array}{ll}131 & 20.7\end{array}$

$137 \quad 23$.

$138 \quad 22.9$

$144 \quad 25.6$

$150 \quad 30.6$

$\begin{array}{ll}158 & 32.9\end{array}$

$235 \quad 122.2$

4842427.0

$332 \quad 302.0$

$360 \quad 416.0$

$379 \quad 496.0$

$\begin{array}{ll}240 & 169.0\end{array}$

$279 \quad 297.0$

$298 \quad 327.0$

$300 \quad 379.0$

$310 \quad 470.0$
$163 \quad 38.4$ $\begin{array}{llll}80.2 & 0.243 & 0.048\end{array}$ $\begin{array}{llll}79.3 & 0.183 & 0.038\end{array}$ $\begin{array}{llll}80 & 0.173 & 0.035\end{array}$ $\begin{array}{llll}80.4 & 0.181 & 0.035\end{array}$ $\begin{array}{llll}79.1 & 0.221 & 0.046\end{array}$ $\begin{array}{llll}79.4 & 0.175 & 0.036\end{array}$ $\begin{array}{llll}79.8 & 0.189 & 0.038\end{array}$ $\begin{array}{llll}79.4 & 0.208 & 0.043\end{array}$ $\begin{array}{llll}78.6 & 0.296 & 0.063\end{array}$

$\begin{array}{llll}75.2 & 2.760 & 0.684\end{array}$ $\begin{array}{llll}76.6 & 0.278 & 0.065\end{array}$ $\begin{array}{llll}75.8 & 0.152 & 0.037\end{array}$ $\begin{array}{llll}77.4 & 0.274 & 0.062\end{array}$ $\begin{array}{llll}78.3 & 0.759 & 0.165\end{array}$ $\begin{array}{llll}78.4 & 1.340 & 0.289\end{array}$ $\begin{array}{llll}76.7 & 1.270 & 0.296\end{array}$

$\begin{array}{llll}78.6 & 1.200 & 0.257\end{array}$

$\begin{array}{llll}77.4 & 1.160 & 0.262\end{array}$
Fillet Fillet Fillet Fillet Fillet Fillet Fillet Fillet Fillet Fillet

Fillet Fillet Fillet Fillet Fillet Fillet Fillet Fillet Fillet
TR-2000-W-002 M TR-2000-W-001 M TR-2000-W-003 M TR-2000-W-004 M TR-2000-W-005 M

TR-2002-F-010 U TR-2002-F-009 U TR-2002-F-008 U TR-2002-F-007 U TR-2002-F-006 U TR-2002-F-004 M TR-2002-F-005 U TR-2002-F-003 M TR-2002-F-002 F TR-2002-F-001 F

TR-2002-F-050 TR-2002-F-049 TR-2002-F-048

TR-2002-F-047 TR-2002-F-046 TR-2002-F-045

TR-2002-F-044 TR-2002-F-043

TR-2002-F-042 TR-2002-F-041

TR-2001-F-314 TR-2001-F-300 TR-2001-F-301 TR-2001-F-302 TR-2001-F-303 TR-2001-F-305 TR-2001-F-306 TR-2001-F-304 TR-2001-F-307

U
F
F
F
F
F
F
F
F
F

F


Table 2. Data for fishes collected in Trinity County, California, 2000-2002 — Continued.

[Fillet samples dissected from left fillet of fish; whole body, whole body fish analyzed with gastrointestinal tract cleaned out. Hwy, highway; Mt, Mount. $\mathrm{Hg}$, mercury; HgT, total mercury. Sex: F, female; M, male; U, unknown. mm, millimeter; g, gram; \%, percent; $\mu \mathrm{g} / \mathrm{g}$, microgram per gram (equivalent to part per million)]

\begin{tabular}{|c|c|c|c|c|c|c|c|c|c|c|}
\hline $\begin{array}{c}\text { Collection } \\
\text { date }\end{array}$ & Common name & Scientific name & $\begin{array}{l}\text { Total } \\
\text { length } \\
\text { (mm) }\end{array}$ & $\begin{array}{l}\text { Total } \\
\text { weight } \\
\text { (g) }\end{array}$ & $\begin{array}{c}\text { Moisture } \\
(\%)\end{array}$ & $\begin{array}{l}\text { HgT- } \\
\text { dry } \\
(\mu \mathrm{g} / \mathrm{g})\end{array}$ & $\begin{array}{c}\text { HgT- } \\
\text { wet } \\
(\mu \mathrm{g} / \mathrm{g})\end{array}$ & Sample type & $\begin{array}{c}\text { Sample } \\
\text { identification }\end{array}$ & $\mathrm{Se}$ \\
\hline \multicolumn{11}{|c|}{ Site 9 (fig. 1), Buckeye Arm of Trinity Lake near Trinity Dam: } \\
\hline $5 / 16 / 2001$ & Largemouth bass & Micropterus salmoides & 219 & 138.0 & 79 & 0.654 & 0.137 & Fillet & TR-2001-F-297 & \\
\hline $5 / 16 / 2001$ & Smallmouth bass & Micropterus dolomieu & 268 & 244.0 & 78.7 & 1.180 & 0.251 & Fillet & TR-2001-F-290 & \\
\hline $5 / 16 / 2001$ & Smallmouth bass & Micropterus dolomieu & 275 & 292.0 & & 1.090 & 0.240 & Fillet & TR-2001-F-294 & \\
\hline $5 / 16 / 2001$ & Smallmouth bas & Micropterus dolomieu & 275 & 298.0 & 78.2 & 0.900 & 0.196 & Fillet & TR-2001-F-291 & \\
\hline $5 / 16 / 2001$ & Smallmouth bass & Micropterus dolomieu & 289 & 310.0 & 78.1 & 1.290 & 0.283 & Fillet & TR-2001-F-292 & \\
\hline $5 / 16 / 2001$ & Smallmouth bass & Micropterus dolomieu & 295 & 350.0 & 77.6 & 0.911 & 0.204 & Fillet & TR-2001-F-293 & \\
\hline $5 / 16 / 2001$ & Smallmouth bass & Micropterus dolomieu & 308 & 360.0 & 78 & 1.220 & 0.268 & Fillet & TR-2001-F-295 & \\
\hline $5 / 16 / 2001$ & Smallmouth bass & Micropterus dolomieu & 329 & 494.0 & 76.7 & 1.150 & 0.268 & Fillet & TR-2001-F-296 & \\
\hline
\end{tabular}

Site 10 (fig. 1), East Fork of Trinity River 0.53 mile above Barney Gulch:

$\begin{array}{ll}8 / 27 / 2002 & \text { Rainbow trout } \\ 8 / 27 / 2002 & \text { Rainbow trout } \\ 8 / 27 / 2002 & \text { Rainbow trout } \\ 8 / 27 / 2002 & \text { Rainbow trout } \\ 8 / 27 / 2002 & \text { Rainbow trout } \\ 8 / 27 / 2002 & \text { Rainbow trout } \\ 8 / 27 / 2002 & \text { Rainbow trout } \\ 8 / 27 / 2002 & \text { Rainbow trout } \\ 8 / 27 / 2002 & \text { Rainbow trout } \\ 8 / 27 / 2002 & \text { Rainbow trout }\end{array}$

Oncorhynchus mykiss 121

Oncorhynchus mykiss

Oncorhynchus mykiss

Oncorhynchus mykiss

Oncorhynchus mykiss

Oncorhynchus mykiss

Oncorhynchus mykiss

Oncorhynchus mykiss

Oncorhynchus mykiss

Oncorhynchus mykiss
Site 11 (fig. 1), New River at Denny Campground near Denny: 8/26/2002 Rainbow trout Oncorhynchus mykiss $8 / 26 / 2002$ Rainbow trout $8 / 26 / 2002$ Rainbow trout $8 / 26 / 2002$ Rainbow trout $8 / 26 / 2002$ Rainbow trout $8 / 26 / 2002$ Rainbow trout 8/26/2002 Rainbow trout $8 / 26 / 2002$ Rainbow trout $8 / 26 / 2002$ Rainbow trout 8/26/2002 Rainbow trout
Oncorhynchus mykiss Oncorhynchus mykiss Oncorhynchus mykiss Oncorhynchus mykiss Oncorhynchus mykiss Oncorhynchus mykiss Oncorhynchus mykiss Oncorhynchus mykiss Oncorhynchus mykiss
16.8

18.2

$126 \quad 19.0$

$\begin{array}{ll}129 & 20.1\end{array}$

$133 \quad 20.8$

$\begin{array}{ll}142 & 26.5\end{array}$

$144 \quad 28.8$

$\begin{array}{ll}155 & 32.1\end{array}$

$\begin{array}{ll}155 & 32.8\end{array}$

$176 \quad 52.1$ $\begin{array}{llll}79.9 & 0.370 & 0.074\end{array}$ $\begin{array}{llll}79.4 & 0.315 & 0.065\end{array}$

$\begin{array}{llll}78.9 & 0.672 & 0.142\end{array}$

$\begin{array}{llll}79.5 & 0.524 & 0.107\end{array}$

$\begin{array}{llll}79.2 & 0.371 & 0.077\end{array}$

$\begin{array}{llll}79.8 & 0.325 & 0.066\end{array}$

$\begin{array}{llll}79.2 & 0.293 & 0.061\end{array}$

$\begin{array}{llll}80 & 0.590 & 0.118\end{array}$

$\begin{array}{llll}79.3 & 0.636 & 0.132\end{array}$

$\begin{array}{llll}79.3 & 0.480 & 0.099\end{array}$
Fillet

Fillet

Fillet

Fillet

Fillet

Fillet

Fillet

Fillet

Fillet

Fillet

\section{$120 \quad 17.5$}

$\begin{array}{ll}125 & 17.9\end{array}$

$137 \quad 25.4$

$149 \quad 28.7$

$155 \quad 36.1$

$156 \quad 33.4$

$\begin{array}{ll}160 & 37.0\end{array}$

$\begin{array}{ll}167 & 42.8\end{array}$

17146.1

20274.0 $\begin{array}{llll}77.4 & 0.232 & 0.052\end{array}$

$\begin{array}{llll}78.9 & 0.307 & 0.065\end{array}$

$\begin{array}{llll}78.4 & 0.300 & 0.065\end{array}$

$\begin{array}{llll}78.6 & 0.235 & 0.050\end{array}$

$\begin{array}{llll}78 & 0.191 & 0.042\end{array}$

$\begin{array}{lll}77.8 & 0.222 & 0.049\end{array}$

$\begin{array}{llll}78.9 & 0.207 & 0.044\end{array}$

$\begin{array}{llll}78.7 & 0.184 & 0.039\end{array}$

$\begin{array}{llll}78.6 & 0.260 & 0.056\end{array}$
Fillet

Fillet

Fillet

Fillet

Fillet

Fillet

Fillet

Fillet

Fillet

Fillet $\begin{array}{lr}\text { TR-2002-F-040 } & \text { F } \\ \text { TR-2002-F-039 } & \text { F } \\ \text { TR-2002-F-038 } & \text { M } \\ \text { TR-2002-F-037 } & \text { F } \\ \text { TR-2002-F-036 } & \text { F } \\ \text { TR-2002-F-035 } & \text { F } \\ \text { TR-2002-F-034 } & \text { F } \\ \text { TR-2002-F-032 } & \text { F } \\ \text { TR-2002-F-033 } & \text { F } \\ \text { TR-2002-F-031 } & \text { M }\end{array}$

TR-2002-F-030

TR-2002-F-029

TR-2002-F-028

TR-2002-F-027

TR-2002-F-026

TR-2002-F-025

TR-2002-F-024

TR-2002-F-023

TR-2002-F-022

TR-2002-F-021

\begin{tabular}{l} 
M \\
\hline \\
$M$ \\
$M$ \\
$M$ \\
$M$ \\
$M$ \\
M \\
\\
F \\
F \\
M \\
F \\
F \\
F \\
F \\
F \\
F \\
M \\
\\
F \\
F \\
F \\
M \\
F \\
F \\
F \\
F \\
F \\
F
\end{tabular}

Site 12 (fig. 1), East Fork Trinity Arm of Trinity Lake near Trinity Center:

$\begin{array}{lllrr}11 / 9 / 2000 & \text { Brown bullhead } & \text { Ameiurus nebulosus } & 360 & 694.6 \\ 11 / 9 / 2000 & \text { Smallmouth bass } & \text { Micropterus dolomieu } & 305 & 495.5 \\ 11 / 9 / 2000 & \text { Smallmouth bass } & \text { Micropterus dolomieu } & 308 & 471.5 \\ 11 / 9 / 2000 & \text { Smallmouth bass } & \text { Micropterus dolomieu } & 310 & 537.5 \\ 11 / 9 / 2000 & \text { Smallmouth bass } & \text { Micropterus dolomieu } & 325 & 583.4 \\ 11 / 9 / 2000 & \text { Smallmouth bass } & \text { Micropterus dolomieu } & 330 & 599.8 \\ 11 / 9 / 2000 & \text { Smallmouth bass } & \text { Micropterus dolomieu } & 349 & 706.1 \\ 11 / 9 / 2000 & \text { Smallmouth bass } & \text { Micropterus dolomieu } & 350 & 683.6 \\ 11 / 9 / 2000 & \text { Smallmouth bass } & \text { Micropterus dolomieu } & 355 & 828.9 \\ 11 / 9 / 2000 & \text { White catfish } & \text { Ameiurus catus } & 325 & 523.5 \\ 11 / 9 / 2000 & \text { White catfish } & \text { Ameiurus catus } & 370 & 712.6 \\ 5 / 15 / 2001 & \text { Brown bullhead } & \text { Ameiurus nebulosus } & 278 & 369.9 \\ 5 / 15 / 2001 & \text { Brown bullhead } & \text { Ameiurus nebulosus } & 330 & 697.9 \\ 5 / 15 / 2001 & \text { Brown bullhead } & \text { Ameiurus nebulosus } & 339 & 733.9 \\ 5 / 15 / 2001 & \text { Largemouth bass } & \text { Micropterus salmoides } & 450 & 1936.4 \\ 5 / 15 / 2001 & \text { Largemouth bass } & \text { Micropterus salmoides } & 489 & 2438.4 \\ 5 / 15 / 2001 & \text { Rainbow trout } & \text { Oncorhynchus mykiss } & 315 & 338.9 \\ 5 / 15 / 2001 & \text { Smallmouth bass } & \text { Micropterus dolomieu } & 188 & 66.9 \\ 5 / 15 / 2001 & \text { Smallmouth bass } & \text { Micropterus dolomieu } & 189 & 70.6 \\ 5 / 15 / 2001 & \text { Smallmouth bass } & \text { Micropterus dolomieu } & 194 & 92.9 \\ 5 / 15 / 2001 & \text { Smallmouth bass } & \text { Micropterus dolomieu } & 206 & 94.9 \\ 5 / 15 / 2001 & \text { Smallmouth bass } & \text { Micropterus dolomieu } & 208 & 113.8\end{array}$

$\begin{array}{lll}78.5 & 0.961 & 0.207\end{array}$

$\begin{array}{llll}75.8 & 1.110 & 0.269\end{array}$

$\begin{array}{lll}76.7 & 1.470 & 0.343\end{array}$

$\begin{array}{llll}75 & 2.160 & 0.540\end{array}$

$\begin{array}{lll}75.9 & 1.370 & 0.330\end{array}$

$\begin{array}{llll}75.6 & 1.330 & 0.325\end{array}$

$\begin{array}{lll}75.9 & 1.960 & 0.472\end{array}$

$\begin{array}{llll}74.7 & 1.480 & 0.374\end{array}$

$\begin{array}{llll}75.9 & 2.100 & 0.506\end{array}$

$\begin{array}{lll}77.5 & 2.620 & 0.590\end{array}$

$\begin{array}{llll}79.7 & 0.693 & 0.141\end{array}$

$\begin{array}{llll}80 & 0.339 & 0.068\end{array}$

$\begin{array}{llll}79.1 & 0.473 & 0.099\end{array}$

$\begin{array}{llll}78.6 & 0.386 & 0.083\end{array}$

$\begin{array}{llll}74.2 & 3.900 & 1.006\end{array}$

$\begin{array}{llll}75.1 & 4.920 & 1.225\end{array}$

$\begin{array}{llll}76 & 0.141 & 0.034\end{array}$

$\begin{array}{llll}79.9 & 1.570 & 0.316\end{array}$

$\begin{array}{llll}79 & 0.788 & 0.165\end{array}$

$\begin{array}{llll}78.8 & 0.558 & 0.118\end{array}$

$\begin{array}{llll}79.3 & 3.840 & 0.795\end{array}$

$\begin{array}{llll}78.7 & 0.808 & 0.172\end{array}$
Fillet

Fillet

Fillet

Fillet

Fillet

Fillet

Fillet

Fillet

Fillet

Fillet

Fillet

Fillet

Fillet

Fillet

Fillet

Fillet

Fillet

Fillet

Fillet

Fillet

Fillet

Fillet
TR-2000-F-248

TR-2000-F-200

TR-2000-F-201

TR-2000-F-202

TR-2000-F-203

TR-2000-F-204

TR-2000-F-205

TR-2000-F-206

TR-2000-F-207

TR-2000-F-247

TR-2000-F-249

TR-2001-F-284

TR-2001-F-285

TR-2001-F-286

TR-2001-F-312

TR-2001-F-313

TR-2001-F-287

TR-2001-F-271

TR-2001-F-270

TR-2001-F-273

TR-2001-F-276

TR-2001-F-280 
Table 2. Data for fishes collected in Trinity County, California, 2000-2002_Continued.

[Fillet samples dissected from left fillet of fish; whole body, whole body fish analyzed with gastrointestinal tract cleaned out. Hwy, highway; Mt, Mount. $\mathrm{Hg}$, mercury; HgT, total mercury. Sex: F, female; M, male; U, unknown. mm, millimeter; g, gram; \%, percent; $\mu \mathrm{g} / \mathrm{g}$, microgram per gram (equivalent to part per million)]

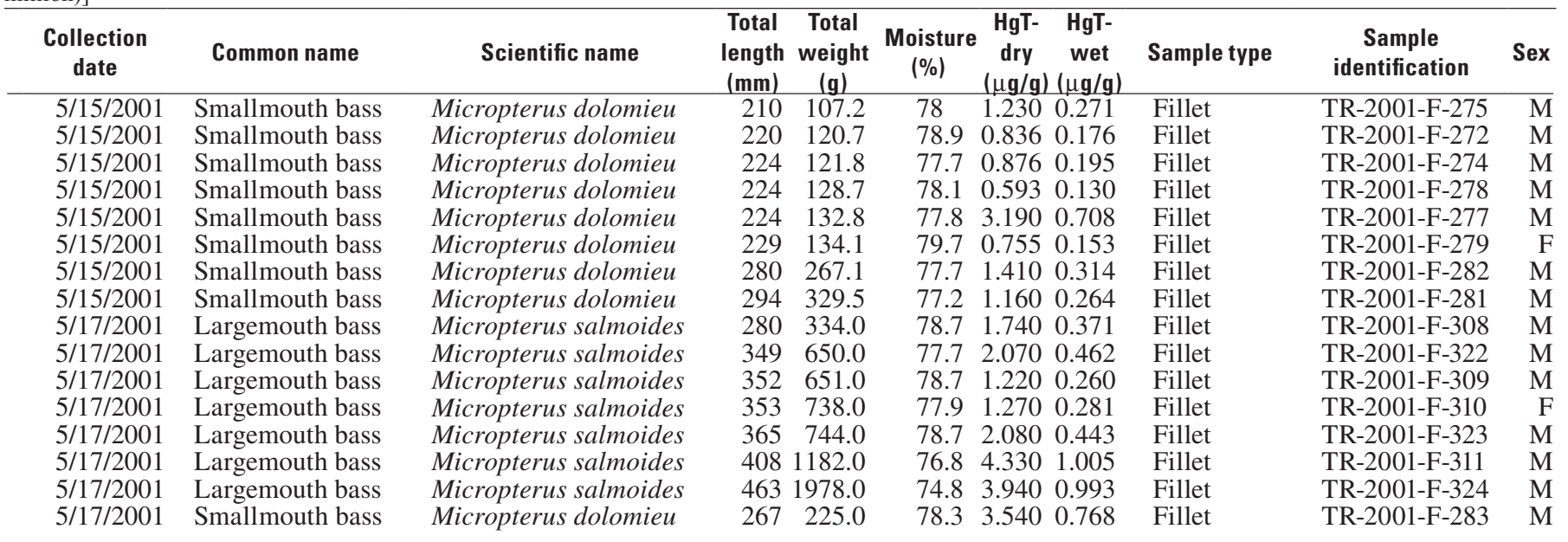

Site 13 (fig. 1), East Fork Trinity River below County Road 106 near Trinity Center: Marbled sculpin 9/6/2001 Marbled sculpin 9/6/2001 Marbled sculpin 9/6/2001 Marbled sculpin 9/6/2001 Marbled sculpin 9/6/2001 Marbled sculpin 9/6/2001 Marbled sculpin 9/6/2001 Marbled sculpin 9/6/2001 Marbled sculpin 9/6/2001 Marbled sculpin 9/6/2001 Marbled sculpin 9/6/2001 Marbled sculpin 9/6/2001 Marbled sculpin 9/6/2001 Rainbow trout 9/6/2001 Rainbow trout 9/6/2001 Rainbow trout 9/6/2001 Rainbow trout 9/6/2001 Rainbow trout 9/6/2001 Rainbow trout $9 / 6 / 2001$ Rainbow trout 9/6/2001 Rainbow trout 9/6/2001 Rainbow trout 8/12/2002 Marbled sculpin 8/12/2002 Marbled sculpin 8/12/2002 Marbled sculpin 8/12/2002 Marbled sculpin 8/12/2002 Marbled sculpin $8 / 12 / 2002$ Rainbow trout $8 / 12 / 2002$ Rainbow trout $8 / 12 / 2002$ Rainbow trout $8 / 12 / 2002$ Rainbow trout $8 / 12 / 2002$ Rainbow trout 8/12/2002 Rainbow trout $8 / 12 / 2002$ Rainbow trout 8/12/2002 Rainbow trout $8 / 12 / 2002$ Rainbow trout $8 / 12 / 2002$ Rainbow trout $8 / 12 / 2002$ Smallmouth bass $8 / 12 / 2002$ Smallmouth bass 8/12/2002 Smallmouth bass $8 / 12 / 2002$ Smallmouth bass 8/12/2002 Smallmouth bass
Cottus klamathensis Cottus klamathensis Cottus klamathensis Cottus klamathensis Cottus klamathensis Cottus klamathensis Cottus klamathensis Cottus klamathensis Cottus klamathensis Cottus klamathensis Cottus klamathensis Cottus klamathensis Cottus klamathensis Cottus klamathensis Oncorhynchus mykiss Oncorhynchus mykiss Oncorhynchus mykiss Oncorhynchus mykiss Oncorhynchus mykiss Oncorhynchus mykiss Oncorhynchus mykiss Oncorhynchus mykiss Oncorhynchus mykiss Cottus klamathensis Cottus klamathensis Cottus klamathensis Cottus klamathensis Cottus klamathensis Oncorhynchus mykiss Oncorhynchus mykiss Oncorhynchus mykiss Oncorhynchus mykiss Oncorhynchus mykiss Oncorhynchus mykiss Oncorhynchus mykiss Oncorhynchus mykiss Oncorhynchus mykiss Micropterus dolomieu Micropterus dolomieu Micropterus dolomieu Micropterus dolomieu Micropterus dolomieu Oncorhynchus mykiss $\begin{array}{lll}75.5 & 0.273 & 0.067\end{array}$ $\begin{array}{llll}73.8 & 0.289 & 0.076\end{array}$ $\begin{array}{llll}74 & 0.273 & 0.071\end{array}$ $\begin{array}{llll}72.3 & 0.312 & 0.086\end{array}$ $\begin{array}{llll}76.2 & 0.277 & 0.066\end{array}$ $\begin{array}{llll}75.9 & 0.463 & 0.112\end{array}$ $\begin{array}{llll}75.2 & 0.198 & 0.049\end{array}$ $\begin{array}{llll}72.4 & 0.253 & 0.070\end{array}$ $\begin{array}{llll}73.7 & 0.215 & 0.057\end{array}$ $\begin{array}{lll}73.5 & 0.247 & 0.065\end{array}$ $\begin{array}{llll}77.7 & 0.621 & 0.138\end{array}$ $\begin{array}{llll}76.2 & 0.661 & 0.157\end{array}$ $\begin{array}{llll}77.8 & 0.476 & 0.106\end{array}$ $\begin{array}{llll}75.8 & 1.030 & 0.249\end{array}$ $\begin{array}{llll}73.6 & 0.372 & 0.098\end{array}$ $\begin{array}{llll}73.9 & 0.444 & 0.116\end{array}$ $\begin{array}{llll}72.6 & 0.567 & 0.155\end{array}$ $\begin{array}{llll}76.6 & 0.379 & 0.089\end{array}$ $\begin{array}{llll}75.5 & 0.429 & 0.105\end{array}$ $\begin{array}{llll}74.9 & 0.357 & 0.090\end{array}$ $\begin{array}{llll}78.2 & 0.313 & 0.068\end{array}$ $\begin{array}{llll}75.1 & 0.560 & 0.139\end{array}$ $\begin{array}{llll}74.8 & 0.618 & 0.156\end{array}$ $\begin{array}{llll}72 & 0.359 & 0.101\end{array}$ $\begin{array}{llll}73.7 & 0.233 & 0.061\end{array}$ $\begin{array}{llll}73.6 & 0.375 & 0.099\end{array}$ $\begin{array}{llll}74.1 & 0.373 & 0.097\end{array}$ $\begin{array}{llll}75.8 & 0.581 & 0.141\end{array}$ $\begin{array}{llll}76.9 & 0.309 & 0.071\end{array}$ $\begin{array}{lll}77.6 & 0.392 & 0.088\end{array}$ $\begin{array}{llll}78.4 & 0.657 & 0.142\end{array}$ $\begin{array}{llll}78 & 0.585 & 0.129\end{array}$ $\begin{array}{llll}78.2 & 0.903 & 0.197\end{array}$ $\begin{array}{llll}78.1 & 0.613 & 0.134\end{array}$ $\begin{array}{llll}78.4 & 0.470 & 0.102\end{array}$ $\begin{array}{llll}78.2 & 0.477 & 0.104\end{array}$ $\begin{array}{llll}77.3 & 1.180 & 0.268\end{array}$ $\begin{array}{llll}76.7 & 1.370 & 0.319\end{array}$ $\begin{array}{lll}75.6 & 0.493 & 0.120\end{array}$ $\begin{array}{llll}78.6 & 1.180 & 0.253\end{array}$ $\begin{array}{llll}78.3 & 0.214 & 0.046\end{array}$ $\begin{array}{llll}77.8 & 0.687 & 0.153\end{array}$ $\begin{array}{lll}78.4 & 0.987 & 0.213\end{array}$
Whole body Whole body Whole body Whole body Whole body Whole body Whole body Whole body Whole body Whole body Fillet

Fillet

Fillet

Fillet

Fillet

Fillet

Fillet

Fillet

Fillet

Fillet

Fillet

Fillet

Fillet

Whole body Whole body Whole body Whole body Whole body Fillet

Fillet

Fillet

Fillet

Fillet

Fillet

Fillet

Fillet

Fillet

Fillet

Fillet

Fillet

Fillet

Fillet

Fillet
TR-2001-W-052 TR-2001-W-051

TR-2001-W-049

TR-2001-W-050

TR-2001-W-048

TR-2001-W-045

TR-2001-W-046

TR-2001-W-047

TR-2001-W-043

TR-2001-W-044

TR-2001-F-346

TR-2001-F-344

TR-2001-F-345

TR-2001-F-343

TR-2001-F-341

TR-2001-F-342

TR-2001-F-340

TR-2001-F-339

TR-2001-F-338

TR-2001-F-337

TR-2001-F-336

TR-2001-F-335

TR-2001-F-334

TR-2002-W-010

TR-2002-W-009

TR-2002-W-008

TR-2002-W-007

TR-2002-W-006

TR-2002-F-102

TR-2002-F-101

TR-2002-F-100

TR-2002-F-099

TR-2002-F-098

TR-2002-F-097

TR-2002-F-096

TR-2002-F-095

TR-2002-F-094

TR-2002-F-093

TR-2002-F-092

TR-2002-F-091

TR-2002-F-090

TR-2002-F-089

TR-2002-F-088
$\mathrm{U}$
$\mathrm{U}$
$\mathrm{U}$
$\mathrm{U}$
$\mathrm{U}$
$\mathrm{U}$
$\mathrm{U}$
$\mathrm{U}$
$\mathrm{U}$
$\mathrm{U}$
$\mathrm{U}$
$\mathrm{U}$
$\mathrm{U}$
$\mathrm{U}$
$\mathrm{U}$
$\mathrm{U}$
$\mathrm{U}$
$\mathrm{U}$
$\mathrm{U}$
$\mathrm{M}$
$\mathrm{M}$
$\mathrm{M}$
$\mathrm{M}$
$\mathrm{F}$
$\mathrm{U}$
$\mathrm{U}$
$\mathrm{U}$
$\mathrm{U}$
$\mathrm{U}$
$\mathrm{U}$
$\mathrm{U}$
$\mathrm{U}$
$\mathrm{U}$
$\mathrm{U}$
$\mathrm{M}$
$\mathrm{M}$
$\mathrm{F}$
$\mathrm{F}$
$\mathrm{U}$
$\mathrm{U}$
$\mathrm{U}$
$\mathrm{U}$
$\mathrm{M}$ 
Table 2. Data for fishes collected in Trinity County, California, 2000-2002 — Continued.

[Fillet samples dissected from left fillet of fish; whole body, whole body fish analyzed with gastrointestinal tract cleaned out. Hwy, highway; Mt, Mount. $\mathrm{Hg}$, mercury; HgT, total mercury. Sex: F, female; M, male; U, unknown. mm, millimeter; g, gram; \%, percent; $\mu \mathrm{g} / \mathrm{g}$, microgram per gram (equivalent to part per million)]

\begin{tabular}{|c|c|c|c|c|c|c|c|c|c|c|}
\hline $\begin{array}{l}\text { Collection } \\
\text { date }\end{array}$ & Common name & Scientific name & $\begin{array}{c}\text { Total } \\
\text { length } \\
\text { (mm) }\end{array}$ & $\begin{array}{c}\text { Total } \\
\text { weight } \\
\text { (g) }\end{array}$ & $\begin{array}{c}\text { Moisture } \\
(\%)\end{array}$ & $\begin{array}{c}\text { HgT- } \\
\text { dry } \\
(\mu \mathrm{g} / \mathrm{g})\end{array}$ & $\begin{array}{c}\text { HgT- } \\
\text { wet } \\
(\mu \mathrm{g} / \mathrm{g})\end{array}$ & Sample type & $\begin{array}{c}\text { Sample } \\
\text { identification }\end{array}$ & Sex \\
\hline \multicolumn{11}{|c|}{ Site 14 (fig. 1), Trinity Lake near Trinity Center: } \\
\hline $10 / 24 / 2000$ & Largemouth bass & Micropterus salmoides & 102 & 16.7 & 75.5 & 0.229 & 0.056 & Whole body & TR-2000-W-026 & $\mathrm{U}$ \\
\hline $10 / 24 / 2000$ & Largemouth bass & Micropterus salmoides & 104 & 18.1 & 76 & 0.210 & 0.050 & Whole body & TR-2000-W-030 & M \\
\hline $10 / 24 / 2000$ & Largemouth bass & Micropterus salmoides & 109 & 19.1 & 74.7 & 0.359 & 0.091 & Whole body & TR-2000-W-028 & M \\
\hline $10 / 24 / 2000$ & Largemouth bass & Micropterus salmoides & 110 & 20.6 & 75.6 & 0.196 & 0.048 & Whole body & TR-2000-W-029 & $\mathrm{U}$ \\
\hline $11 / 9 / 2000$ & Green sunfish & Lepomis cyanellus & 180 & 113.7 & 80.7 & 0.830 & 0.160 & Fillet & TR-2000-F-213 & M \\
\hline $11 / 9 / 2000$ & Largemouth bass & Micropterus salmoides & 235 & 185.8 & 79.7 & 0.584 & 0.119 & Fillet & TR-2000-F-219 & $\mathrm{F}$ \\
\hline $11 / 9 / 2000$ & Rainbow trout & Oncorhynchus mykiss & 194 & 65.3 & 77.4 & 0.143 & 0.032 & Fillet & TR-2000-F-211 & $\mathrm{U}$ \\
\hline $11 / 9 / 2000$ & Rainbow trout & Oncorhynchus mykiss & 238 & 128.8 & 78.6 & 0.311 & 0.067 & Fillet & TR-2000-F-210 & M \\
\hline $11 / 9 / 2000$ & Rainbow trout & Oncorhynchus mykiss & 295 & 248.3 & 79.3 & 0.573 & 0.119 & Fillet & TR-2000-F-215 & M \\
\hline $11 / 9 / 2000$ & Rainbow trout & Oncorhynchus mykiss & 309 & 302.4 & 77 & 0.457 & 0.105 & Fillet & TR-2000-F-217 & M \\
\hline $11 / 9 / 2000$ & Rainbow trout & Oncorhynchus mykiss & - & 215.5 & 76.5 & 0.362 & 0.085 & Fillet & TR-2000-F-218 & M \\
\hline $5 / 15 / 2001$ & Smallmouth bass & Micropterus dolomieu & 172 & 56.2 & 79.9 & 1.070 & 0.215 & Fillet & TR-2001-F-289 & $\mathrm{F}$ \\
\hline $5 / 15 / 2001$ & Smallmouth bass & Micropterus dolomieu & 181 & 72.5 & 80.2 & 1.200 & 0.238 & Fillet & TR-2001-F-288 & M \\
\hline $5 / 17 / 2001$ & Largemouth bass & Micropterus salmoides & 260 & 248.3 & 78.3 & 1.880 & 0.408 & Fillet & TR-2001-F-315 & M \\
\hline $5 / 17 / 2001$ & Largemouth bass & Micropterus salmoides & 303 & 487.8 & 77.5 & 0.993 & 0.223 & Fillet & TR-2001-F-316 & $\mathrm{F}$ \\
\hline $5 / 17 / 2001$ & Largemouth bass & Micropterus salmoides & 307 & 492.6 & 77.9 & 1.130 & 0.250 & Fillet & TR-2001-F-320 & M \\
\hline $5 / 17 / 2001$ & Largemouth bass & Micropterus salmoides & 310 & 507.1 & 76.6 & 1.280 & 0.300 & Fillet & TR-2001-F-317 & M \\
\hline $5 / 17 / 2001$ & Largemouth bass & Micropterus salmoides & 310 & 655.1 & 76.9 & 1.460 & 0.337 & Fillet & TR-2001-F-318 & $\mathrm{F}$ \\
\hline $5 / 17 / 2001$ & Largemouth bass & Micropterus salmoides & 315 & 593.2 & 76.5 & 1.220 & 0.287 & Fillet & TR-2001-F-319 & M \\
\hline $5 / 17 / 2001$ & Largemouth bass & Micropterus salmoides & 350 & 705.1 & 77.4 & 1.580 & 0.357 & Fillet & TR-2001-F-321 & $\mathrm{F}$ \\
\hline $5 / 17 / 2001$ & Largemouth bass & Micropterus salmoides & 355 & 864.8 & 76.6 & 1.690 & 0.395 & Fillet & TR-2001-F-325 & $\mathrm{F}$ \\
\hline $5 / 17 / 2001$ & Largemouth bass & Micropterus salmoides & 358 & 718.2 & 77.1 & 3.210 & 0.735 & Fillet & TR-2001-F-326 & M \\
\hline $5 / 17 / 2001$ & Largemouth bass & Micropterus salmoides & 385 & 976.9 & 75.3 & 2.290 & 0.566 & Fillet & TR-2001-F-329 & M \\
\hline 9/6/2001 & Rainbow trout & Oncorhynchus mykiss & 103 & 9.7 & 76.6 & 0.626 & 0.146 & Fillet & TR-2001-F-413 & $\mathrm{U}$ \\
\hline $9 / 6 / 2001$ & Rainbow trout & Oncorhynchus mykiss & 108 & 11.2 & 76.5 & 0.719 & 0.169 & Fillet & TR-2001-F-412 & $\mathrm{U}$ \\
\hline $9 / 6 / 2001$ & Rainbow trout & Oncorhynchus mykiss & 113 & 13.0 & 75.1 & 0.666 & 0.166 & Fillet & TR-2001-F-411 & $\mathrm{U}$ \\
\hline 9/6/2001 & Rainbow trout & Oncorhynchus mykiss & 119 & 14.9 & 78.5 & 0.617 & 0.133 & Fillet & TR-2001-F-410 & $\mathrm{U}$ \\
\hline $9 / 6 / 2001$ & Rainbow trout & Oncorhynchus mykiss & 142 & 24.7 & 78.3 & 0.732 & 0.159 & Fillet & TR-2001-F-408 & M \\
\hline $9 / 6 / 2001$ & Rainbow trout & Oncorhynchus mykiss & 148 & 28.0 & 75.6 & 0.719 & 0.175 & Fillet & TR-2001-F-407 & M \\
\hline $9 / 6 / 2001$ & Rainbow trout & Oncorhynchus mykiss & 165 & 41.8 & 77.1 & 1.140 & 0.261 & Fillet & TR-2001-F-402 & $\mathrm{U}$ \\
\hline $9 / 6 / 2001$ & Rainbow trout & Oncorhynchus mykiss & 168 & 41.4 & 78.7 & 0.833 & 0.177 & Fillet & TR-2001-F-403 & M \\
\hline $9 / 6 / 2001$ & Rainbow trout & Oncorhynchus mykiss & 196 & 66.1 & 77.5 & 0.716 & 0.161 & Fillet & TR-2001-F-400 & M \\
\hline 9/6/2001 & Rainbow trout & Oncorhynchus mykiss & 220 & 117.8 & 77.3 & 1.810 & 0.411 & Fillet & TR-2001-F-399 & M \\
\hline $8 / 12 / 2002$ & Marbled sculpin & Cottus klamathensis & 63 & 3.1 & 76.2 & 0.525 & 0.125 & Whole body & TR-2002-W-004 & $\mathrm{U}$ \\
\hline $8 / 12 / 2002$ & Marbled sculpin & Cottus klamathensis & 63 & 3.1 & 75.9 & 0.967 & 0.233 & Whole body & TR-2002-W-005 & $\mathrm{U}$ \\
\hline $8 / 12 / 2002$ & Marbled sculpin & Cottus klamathensis & 65 & 2.7 & 75.4 & 0.441 & 0.108 & Whole body & TR-2002-W-002 & $\mathrm{U}$ \\
\hline $8 / 12 / 2002$ & Marbled sculpin & Cottus klamathensis & 65 & 3.3 & 76.4 & 0.738 & 0.174 & Whole body & TR-2002-W-003 & $\mathrm{U}$ \\
\hline $8 / 12 / 2002$ & Marbled sculpin & Cottus klamathensis & 66 & 3.1 & 73.5 & 0.515 & 0.136 & Whole body & TR-2002-W-001 & $\mathrm{U}$ \\
\hline $8 / 12 / 2002$ & Rainbow trout & Oncorhynchus mykiss & 121 & 15.4 & 79.1 & 0.733 & 0.153 & Fillet & TR-2002-F-086 & $\mathrm{U}$ \\
\hline $8 / 12 / 2002$ & Rainbow trout & Oncorhynchus mykiss & 121 & 15.8 & 80.6 & 0.911 & 0.177 & Fillet & TR-2002-F-087 & $\mathrm{U}$ \\
\hline $8 / 12 / 2002$ & Rainbow trout & Oncorhynchus mykiss & 129 & 19.1 & 80.7 & 0.622 & 0.120 & Fillet & TR-2002-F-085 & $\mathrm{U}$ \\
\hline $8 / 12 / 2002$ & Rainbow trout & Oncorhynchus mykiss & 137 & 23.5 & 79.1 & 0.970 & 0.203 & Fillet & TR-2002-F-084 & M \\
\hline $8 / 12 / 2002$ & Rainbow trout & Oncorhynchus mykiss & 161 & 35.4 & 79.5 & 0.840 & 0.172 & Fillet & TR-2002-F-083 & $\mathrm{F}$ \\
\hline $8 / 12 / 2002$ & Rainbow trout & Oncorhynchus mykiss & 165 & 40.5 & 79.1 & 0.752 & 0.157 & Fillet & TR-2002-F-082 & $\mathrm{F}$ \\
\hline
\end{tabular}


Table 2. Data for fishes collected in Trinity County, California, 2000-2002_Continued.

[Fillet samples dissected from left fillet of fish; whole body, whole body fish analyzed with gastrointestinal tract cleaned out. Hwy, highway; Mt, Mount. $\mathrm{Hg}$, mercury; HgT, total mercury. Sex: F, female; M, male; $\mathrm{U}$, unknown. mm, millimeter; g, gram; \%, percent; $\mu \mathrm{g} / \mathrm{g}$, microgram per gram (equivalent to part per million)]

\begin{tabular}{|c|c|c|c|c|c|c|c|c|c|}
\hline $\begin{array}{l}\text { Collection } \\
\text { date }\end{array}$ & Common name & Scientific name & $\begin{array}{l}\text { Total } \\
\text { length } \\
\text { (mm) }\end{array}$ & $\begin{array}{c}\text { Total } \\
\text { weight } \\
\text { (g) }\end{array}$ & $\begin{array}{c}\text { Moisture } \\
(\%)\end{array}$ & $\begin{array}{c}\text { HgT- } \\
\text { dry } \\
(\mu \mathrm{g} / \mathrm{g})\end{array}$ & $\begin{array}{c}\text { HgT- } \\
\text { wet } \\
(\mu \mathrm{g} / \mathrm{g})\end{array}$ & Sample type & $\begin{array}{c}\text { Sample } \\
\text { identification }\end{array}$ \\
\hline $8 / 12 / 2002$ & Rainbow trout & Oncorhynchus mykiss & 170 & 40.2 & 79.4 & 0.534 & 0.110 & Fillet & TR-2002-F-081 \\
\hline $8 / 12 / 2002$ & Rainbow trout & Oncorhynchus mykiss & 194 & 66.4 & 78.5 & 0.730 & 0.157 & Fillet & TR-2002-F-080 \\
\hline $8 / 12 / 2002$ & Rainbow trout & Oncorhynchus mykiss & 216 & 92.2 & 78.9 & 1.120 & 0.236 & Fillet & TR-2002-F-078 \\
\hline
\end{tabular}

Site 16 (fig. 1), Carrville Pond near Carrville:

\begin{tabular}{|c|c|c|}
\hline $9 / 12 / 2000$ & Rainbow trout & Oncorhynchus mykiss \\
\hline $9 / 26 / 2000$ & Rainbow trout & Oncorhynchus mykiss \\
\hline $9 / 26 / 2000$ & Rainbow trout & Oncorhynchus mykiss \\
\hline $9 / 26 / 2000$ & Rainbow trout & Oncorhynchus mykiss \\
\hline $9 / 26 / 2000$ & Rainbow trout & Oncorhynchus mykiss \\
\hline $9 / 26 / 2000$ & Rainbow trout & Oncorhynchus mykiss \\
\hline $9 / 26 / 2000$ & Rainbow trout & Oncorhynchus mykiss \\
\hline & Rainbow trout & Oncorhynchus mykiss \\
\hline & Rainbow trout & Oncorhynchus mykiss \\
\hline & Rainbow trout & Oncorhynchus mykiss \\
\hline
\end{tabular}

$\begin{array}{ll}276 & 189.6 \\ 260 & 209.3 \\ 280 & 262.2 \\ 282 & 204.6 \\ 283 & 275.9 \\ 292 & 248.6 \\ 296 & 328.3 \\ 300 & 272.8 \\ 315 & 319.6 \\ 325 & 355.4\end{array}$

$\begin{array}{llll}75.3 & 0.096 & 0.024\end{array}$

$\begin{array}{lll}76.8 & 0.092 & 0.021\end{array}$

$\begin{array}{lll}76.4 & 0.109 & 0.026\end{array}$

$\begin{array}{lll}75.6 & 0.099 & 0.024\end{array}$

$\begin{array}{llll}74.7 & 0.089 & 0.023\end{array}$

$\begin{array}{llll}75 & 0.086 & 0.021\end{array}$

$\begin{array}{llll}75.9 & 0.078 & 0.019\end{array}$

$\begin{array}{llll}75.5 & 0.089 & 0.022\end{array}$

$\begin{array}{lll}75.6 & 0.126 & 0.031\end{array}$

$135 \quad 24.0$

$\begin{array}{ll}154 & 34.5\end{array}$

$163 \quad 45.0$

$\begin{array}{ll}170 & 46.9\end{array}$

$\begin{array}{ll}177 & 52.4\end{array}$

$206 \quad 83.6$

$218 \quad 105.3$

$\begin{array}{ll}229 & 125.7\end{array}$

$\begin{array}{ll}242 & 145.8\end{array}$

$358 \quad 390.9$

Oncorhynchus mykiss

Oncorhynchus mykiss $\begin{array}{llll}74.8 & 0.126 & 0.032\end{array}$

$\begin{array}{llll}73.8 & 0.096 & 0.025\end{array}$

$\begin{array}{llll}74.6 & 0.152 & 0.039\end{array}$

$\begin{array}{llll}73.4 & 0.080 & 0.021\end{array}$

$\begin{array}{lll}75.3 & 0.081 & 0.020\end{array}$

$\begin{array}{llll}77 & 0.141 & 0.032\end{array}$

$\begin{array}{llll}77.4 & 0.819 & 0.185\end{array}$

$\begin{array}{llll}77.6 & 0.184 & 0.041\end{array}$

$\begin{array}{llll}75.9 & 0.165 & 0.040\end{array}$

$\begin{array}{llll}75.7 & 0.410 & 0.100\end{array}$
Fillet

Fillet

Fillet

Fillet

Fillet

Fillet

Fillet

Fillet

Fillet

Fillet

Whole body Whole body Whole body Whole body Whole body Fillet

Fillet

Fillet

Fillet

Fillet

Whole body Whole body Whole body Whole body Whole body Fillet

Fillet

Fillet

Fillet

Fillet $\begin{array}{ll}\text { TR-2000-F-229 } & \text { M } \\ \text { TR-2000-F-220 } & \text { M } \\ \text { TR-2000-F-223 } & M \\ \text { TR-2000-F-221 } & \text { M } \\ \text { TR-2000-F-222 } & \text { M } \\ \text { TR-2000-F-226 } & \text { M } \\ \text { TR-2000-F-227 } & \text { M } \\ \text { TR-2000-F-225 } & \text { M } \\ \text { TR-2000-F-224 } & \text { M } \\ \text { TR-2000-F-228 } & \text { M }\end{array}$

TR-2000-W-006 U TR-2000-W-007 M TR-2000-W-010 M TR-2000-W-008 M TR-2000-W-009 U TR-2000-F-236 M TR-2000-F-237 M TR-2000-F-238 F TR-2000-F-239 F TR-2000-F-240 F

TR-2000-W-012 U TR-2000-W-013 M TR-2000-W-011 M TR-2000-W-014 M TR-2000-W-015 U TR-2000-F-241 M TR-2000-F-242 M TR-2000-F-244 M TR-2000-F-243 M TR-2000-F-245 M

Whole body Whole body Whole body Whole body Whole body

Fillet

Fillet

Fillet

Fillet

Fillet

Fillet

Fillet

Fillet

Fillet

Fillet

Fillet

Fillet
TR-2000-W-023 M TR-2000-W-024 M TR-2000-W-025 U TR-2000-W-022 F TR-2000-W-021 F TR-2000-F-230 F TR-2000-F-232 M TR-2000-F-231 F TR-2001-F-398 U TR-2001-F-397 U TR-2001-F-394 M TR-2001-F-392 M TR-2001-F-390 U TR-2001-F-389 U TR-2001-F-388 F TR-2001-F-386 M TR-2001-F-384 M 
Table 2. Data for fishes collected in Trinity County, California, 2000-2002 — Continued.

[Fillet samples dissected from left fillet of fish; whole body, whole body fish analyzed with gastrointestinal tract cleaned out. Hwy, highway; Mt, Mount. $\mathrm{Hg}$, mercury; HgT, total mercury. Sex: F, female; M, male; U, unknown. mm, millimeter; g, gram; \%, percent; $\mu \mathrm{g} / \mathrm{g}$, microgram per gram (equivalent to part per million)]

\begin{tabular}{|c|c|c|c|c|c|c|c|c|c|c|}
\hline $\begin{array}{l}\text { Collection } \\
\text { date }\end{array}$ & Common name & Scientific name & $\begin{array}{l}\text { Total } \\
\text { length } \\
\text { (mm) }\end{array}$ & $\begin{array}{l}\text { Total } \\
\text { weight } \\
\text { (g) }\end{array}$ & $\begin{array}{c}\text { Moisture } \\
(\%)\end{array}$ & $\begin{array}{c}\text { HgT- } \\
\text { dry } \\
(\mu \mathrm{g} / \mathrm{g})\end{array}$ & $\begin{array}{c}\text { HgT- } \\
\text { wet } \\
(\mu \mathrm{g} / \mathrm{g})\end{array}$ & Sample type & $\begin{array}{c}\text { Sample } \\
\text { identification }\end{array}$ & Sex \\
\hline 9/5/2001 & Rainbow trout & Oncorhynchus mykiss & 154 & 31.6 & 77.1 & 0.822 & 0.188 & Fillet & TR-2001-F-385 & $\bar{F}$ \\
\hline $8 / 13 / 2002$ & Rainbow trout & Oncorhynchus mykiss & 130 & 23.6 & 80.1 & 0.901 & 0.179 & Fillet & TR-2002-F-112 & $\mathrm{U}$ \\
\hline $8 / 13 / 2002$ & Rainbow trout & Oncorhynchus mykiss & 133 & 21.9 & 79.8 & 0.853 & 0.172 & Fillet & TR-2002-F-111 & $\mathrm{U}$ \\
\hline $8 / 13 / 2002$ & Rainbow trout & Oncorhynchus mykiss & 134 & 23.0 & 80 & 0.872 & 0.174 & Fillet & TR-2002-F-109 & $\mathrm{U}$ \\
\hline $8 / 13 / 2002$ & Rainbow trout & Oncorhynchus mykiss & 134 & 22.2 & 79.2 & 1.610 & 0.335 & Fillet & TR-2002-F-110 & $\mathrm{U}$ \\
\hline $8 / 13 / 2002$ & Rainbow trout & Oncorhynchus mykiss & 137 & 25.0 & 79.7 & 0.924 & 0.188 & Fillet & TR-2002-F-108 & M \\
\hline $8 / 13 / 2002$ & Rainbow trout & Oncorhynchus mykiss & 155 & 36.3 & 79.4 & 1.100 & 0.227 & Fillet & TR-2002-F-106 & $\mathrm{F}$ \\
\hline $8 / 13 / 2002$ & Rainbow trout & Oncorhynchus mykiss & 156 & 39.5 & 79.8 & 1.110 & 0.224 & Fillet & TR-2002-F-104 & $\mathrm{F}$ \\
\hline $8 / 13 / 2002$ & Rainbow trout & Oncorhynchus mykiss & 160 & 41.6 & 80.4 & 0.670 & 0.131 & Fillet & TR-2002-F-103 & $\mathrm{F}$ \\
\hline
\end{tabular}

Site 20 (fig. 1), East Fork Trinity River Upstream Altoona Mine Drain near Castella: 9/11/2000 Rainbow trout 9/11/2000 Rainbow trout 9/11/2000 Rainbow trout 9/11/2000 Rainbow trout $9 / 11 / 2000$ Rainbow trout 9/11/2000 Rainbow trout 9/11/2000 Rainbow trout 9/11/2000 Rainbow trout 9/5/2001 Rainbow trout 9/5/2001 Rainbow trout 9/5/2001 Rainbow trout $9 / 5 / 2001$ Rainbow trout 9/5/2001 Rainbow trout $9 / 5 / 2001$ Rainbow trout 9/5/2001 Rainbow trout 9/5/2001 Rainbow trout $9 / 5 / 2001$ Rainbow trout 9/5/2001 Rainbow trout 8/13/2002 Rainbow trout $8 / 13 / 2002$ Rainbow trout $8 / 13 / 2002$ Rainbow trout 8/13/2002 Rainbow trout 8/13/2002 Rainbow trout $8 / 13 / 2002$ Rainbow trout $8 / 13 / 2002$ Rainbow trout $8 / 13 / 2002$ Rainbow trout $8 / 13 / 2002$ Rainbow trout 8/13/2002 Rainbow trout Oncorhynchus mykiss Oncorhynchus mykiss Oncorhynchus mykiss Oncorhynchus mykiss Oncorhynchus mykiss Oncorhynchus mykiss Oncorhynchus mykiss Oncorhynchus mykiss Oncorhynchus mykiss Oncorhynchus mykiss Oncorhynchus mykiss Oncorhynchus mykiss Oncorhynchus mykiss Oncorhynchus mykiss Oncorhynchus mykiss Oncorhynchus mykiss Oncorhynchus mykiss Oncorhynchus mykiss Oncorhynchus mykiss Oncorhynchus mykiss Oncorhynchus mykiss Oncorhynchus mykiss Oncorhynchus mykiss Oncorhynchus mykiss Oncorhynchus mykiss Oncorhynchus mykiss Oncorhynchus mykiss Oncorhynchus mykiss
12118.

$\begin{array}{ll}133 & 22.8\end{array}$

$139 \quad 26.5$

$142 \quad 27.6$

$148 \quad 28.2$

$\begin{array}{ll}189 & 71.8\end{array}$

$198 \quad 82.7$

$200 \quad 76.1$

$90 \quad 7.8$

$\begin{array}{ll}94 & 6.7\end{array}$

$106 \quad 11.3$

$110 \quad 12.1$

$134 \quad 21.2$

$138 \quad 23.7$

$\begin{array}{ll}140 & 27.3\end{array}$

$148 \quad 28.8$

$\begin{array}{ll}165 & 43.6\end{array}$

$133 \quad 23.0$

$\begin{array}{ll}134 & 23.0\end{array}$

$\begin{array}{ll}136 & 25.9\end{array}$

$138 \quad 25.7$

$142 \quad 26.5$

$144 \quad 31.0$

$\begin{array}{ll}152 & 35.7\end{array}$

16240.0

$175 \quad 58.3$
$108 \quad 10.5$

$141 \quad 29.1$ $\begin{array}{llll}73.6 & 0.509 & 0.134\end{array}$ $\begin{array}{llll}75 & 0.462 & 0.116\end{array}$ $\begin{array}{llll}77.1 & 0.444 & 0.102\end{array}$ $\begin{array}{llll}76.6 & 0.388 & 0.091\end{array}$ $\begin{array}{llll}78 & 0.542 & 0.119\end{array}$ $\begin{array}{llll}76.8 & 1.230 & 0.285\end{array}$ $\begin{array}{llll}75.8 & 0.943 & 0.228\end{array}$ $\begin{array}{llll}77.4 & 1.120 & 0.253\end{array}$ $\begin{array}{llll}77 & 0.565 & 0.130\end{array}$ $\begin{array}{llll}76.5 & 0.351 & 0.082\end{array}$ $\begin{array}{llll}73.9 & 0.548 & 0.143\end{array}$ $\begin{array}{llll}77.7 & 0.554 & 0.124\end{array}$ $\begin{array}{llll}77.3 & 0.563 & 0.128\end{array}$ $\begin{array}{llll}75.9 & 0.790 & 0.190\end{array}$ $\begin{array}{llll}75.8 & 0.528 & 0.128\end{array}$ $\begin{array}{llll}75.2 & 0.748 & 0.186\end{array}$ $\begin{array}{llll}76.1 & 0.725 & 0.173\end{array}$ $\begin{array}{llll}75.2 & 0.716 & 0.178\end{array}$ $\begin{array}{llll}80.1 & 0.893 & 0.178\end{array}$ $\begin{array}{llll}80.6 & 0.660 & 0.128\end{array}$ $\begin{array}{llll}79.8 & 0.708 & 0.143\end{array}$ $\begin{array}{llll}79.3 & 0.774 & 0.160\end{array}$ $\begin{array}{llll}78.8 & 0.591 & 0.125\end{array}$ $\begin{array}{llll}79.3 & 1.150 & 0.238\end{array}$ $\begin{array}{llll}79.1 & 0.673 & 0.141\end{array}$ $\begin{array}{llll}80.4 & 1.110 & 0.218\end{array}$ $\begin{array}{llll}79.7 & 0.849 & 0.172\end{array}$ $\begin{array}{llll}79.3 & 0.696 & 0.144\end{array}$
Whole body Whole body Whole body Whole body Whole body Fillet Fillet Fillet Fillet Fillet Fillet Fillet Fillet Fillet Fillet Fillet Fillet Fillet Fillet Fillet Fillet Fillet Fillet Fillet Fillet Fillet Fillet Fillet

$\begin{array}{lll}76.4 & 0.369 & 0.087\end{array}$ $\begin{array}{lll}78.3 & 0.553 & 0.120\end{array}$ $\begin{array}{llll}73.1 & 0.461 & 0.124\end{array}$ $\begin{array}{llll}75.9 & 0.340 & 0.082\end{array}$ $\begin{array}{llll}78.2 & 0.700 & 0.153\end{array}$ $\begin{array}{llll}76.4 & 0.530 & 0.125\end{array}$ $\begin{array}{llll}77.3 & 0.619 & 0.141\end{array}$ $\begin{array}{llll}77.6 & 0.768 & 0.172\end{array}$ $\begin{array}{lll}77 & 0.509 & 0.117\end{array}$ $\begin{array}{llll}77.4 & 1.210 & 0.273\end{array}$ $\begin{array}{llll}79.2 & 0.577 & 0.120\end{array}$ $\begin{array}{llll}80 & 0.741 & 0.148\end{array}$ $\begin{array}{llll}79.2 & 0.545 & 0.113\end{array}$ $\begin{array}{llll}79.4 & 0.585 & 0.121\end{array}$ $\begin{array}{llll}79.6 & 0.572 & 0.117\end{array}$ $\begin{array}{llll}79.7 & 0.578 & 0.117\end{array}$
Fillet Fillet Fillet Fillet Fillet Fillet Fillet Fillet Fillet Fillet Fillet Fillet Fillet Fillet Fillet Fillet
TR-2000-W-017 M TR-2000-W-018 M TR-2000-W-016 M TR-2000-W-019 M TR-2000-W-020 M TR-2000-F-235 F TR-2000-F-233 M TR-2000-F-234 F TR-2001-F-382 U TR-2001-F-383 U TR-2001-F-380 U TR-2001-F-381 M TR-2001-F-379 U TR-2001-F-378 F TR-2001-F-377 F TR-2001-F-376 M TR-2001-F-374 M TR-2001-F-373 F TR-2002-F-077 TR-2002-F-076 TR-2002-F-075

TR-2002-F-074

TR-2002-F-073

TR-2002-F-072

TR-2002-F-071

TR-2002-F-070

TR-2002-F-069 TR-2002-F-068

TR-2001-F-427 TR-2001-F-428

TR-2001-F-426

TR-2001-F-425

TR-2001-F-422

TR-2001-F-423

TR-2001-F-420

TR-2001-F-421

TR-2001-F-417

TR-2001-F-414

TR-2002-F-122

TR-2002-F-121

TR-2002-F-120

TR-2002-F-119

TR-2002-F-118

TR-2002-F-117
$8 / 13 / 2002$ Rainbow trout

$8 / 13 / 2002$ Rainbow trout
$143 \quad 32.9$

$\begin{array}{ll}150 & 31.9\end{array}$
Oncorhynchus mykis

F


Table 2. Data for fishes collected in Trinity County, California, 2000-2002_Continued.

[Fillet samples dissected from left fillet of fish; whole body, whole body fish analyzed with gastrointestinal tract cleaned out. Hwy, highway; Mt, Mount. $\mathrm{Hg}$, mercury; HgT, total mercury. Sex: F, female; M, male; $\mathrm{U}$, unknown. mm, millimeter; g, gram; \%, percent; $\mu \mathrm{g} / \mathrm{g}$, microgram per gram (equivalent to part per million)]

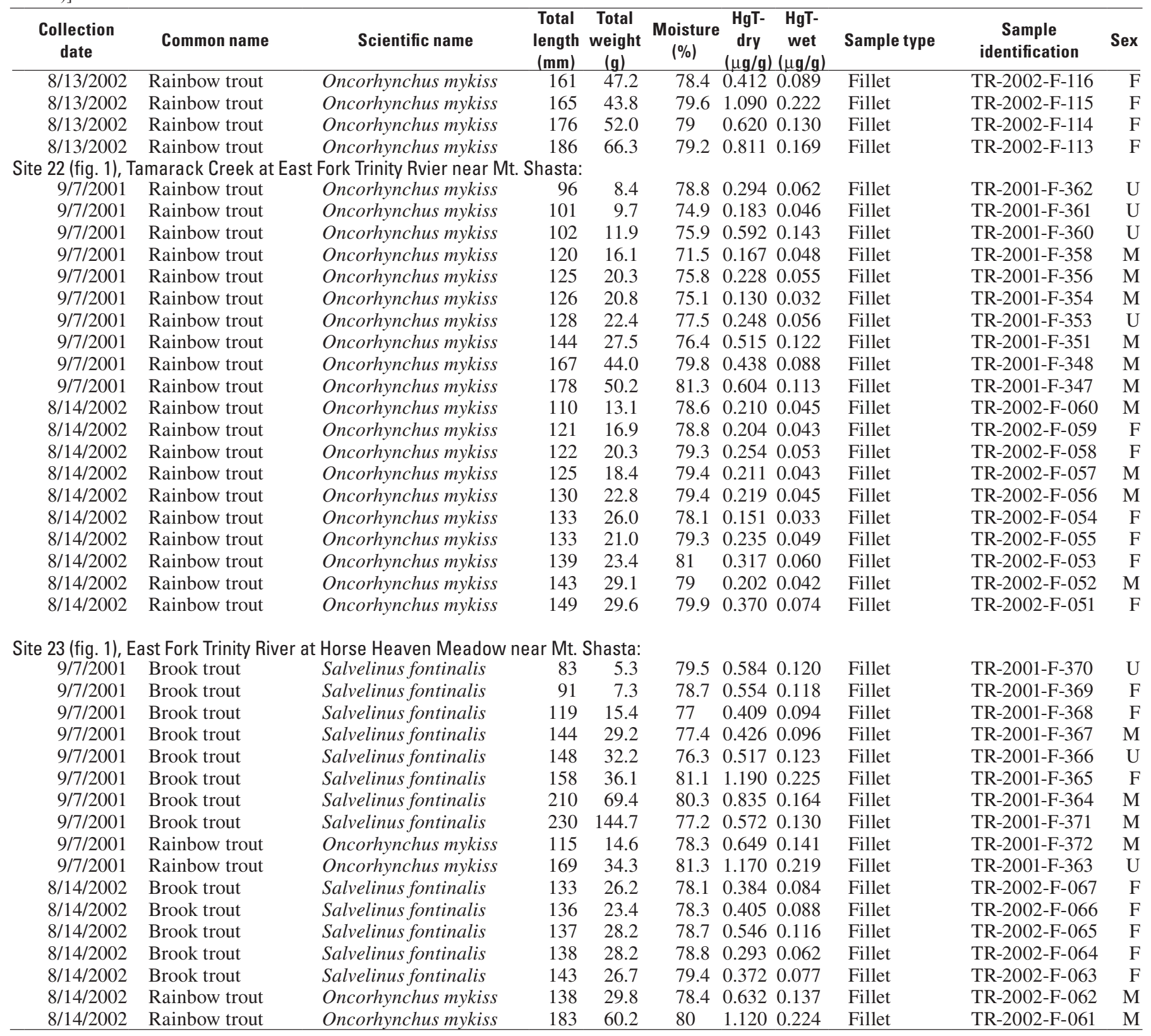




\section{Field Methods and Sample Preservation Techniques}

Field and laboratory methods were similar to those used by May and others (2000). Most fish were collected using electrofishing equipment and dip nets. Additional collection techniques included gill netting, hook and line capture, as well as dip netting. Fish were held in clean containers with ambient water until they were weighed, to the nearest gram, and measured for standard and total length, in millimeters. After recording the length and mass, spines or scales were removed for future age determination. Each fish was then wrapped in clean, heavy-duty aluminum foil, labeled, placed in a plastic bag on wet ice and held for less than 8 hours. The fish were then taken to the laboratory where they were stored frozen until processing.

The processing of fish followed standard procedures (U.S. Environmental Protection Agency, 1995). Fish were handled with powder-free latex gloves, and dissections were performed on a new sheet of heavy-duty aluminum foil for each fish. High-quality stainless steel instruments and disposable scalpel blades were used in the processing of the fish samples. Scalpel blades were changed and instruments were cleaned thoroughly between samples. Cleaning the instruments involved washing with deionized water and laboratory detergent, acid washing, and finally rinsing with deionized water before and after dissection of each fish specimen.

Larger fish were thawed and scaled or the skin was removed (on scaleless fish such as catfish) before dissection. Boneless and skinless fillet portions were dissected from the upper medial-axial region of the fish in an approximately rectangular shape. Excised tissues were placed directly into labeled, chemically cleaned borosilicate-glass jars on a pre-tared balance. The sample mass was recorded, and a Teflon-lined lid was screwed atop each jar and sealed with Parafilm. Fish tissue samples were stored frozen in sealed sample jars until they were packed in coolers with dry ice and shipped to the analytical laboratory.

Muscle tissues were removed from both the left and right fillet of the larger fish processed during this study. Tissues dissected from the left fillet were labeled with sample numbers beginning with "F-" for individual samples. Tissues removed from the right fillet were labeled with sample numbers beginning with "R-"; these samples served as archive samples, as well as replicates for additional quality-assurance purposes.

For larger fish (longer than about 175-mm total length), concentrations of total mercury $(\mathrm{Hg})$ were determined from fillet samples of axial muscle. For some smaller fish, such as marbled sculpin (Cottus klamathensis), rainbow trout (Oncorhynchus mykiss), and green sunfish (Lepomis cyanellus), whole-body samples (with the gastrointestinal contents removed) were submitted for total mercury analysis.

\section{Laboratory Methods of Chemical Analysis}

Analysis of mercury $(\mathrm{Hg})$ in fish samples was done at the Trace Element Research Laboratory (TERL) at Texas A\&M University in College Station, Texas, under the direction of Dr. Robert Taylor. Before samples were analyzed for $\mathrm{Hg}$ by cold-vapor atomic absorption spectroscopy (CVAAS), the $\mathrm{Hg}$ was converted to the divalent mercury $\left(\mathrm{Hg}^{2+}\right)$ form. Mercury was digested using a modified version of U.S. Environmental Protection Agency (USEPA) methods 245.5 and 245.6 (U.S. Environmental Protection Agency, 1991). Tissue samples were homogenized in the original sample containers using a Tekmar Tissumizer, and then subsampled. Tissue subsamples were digested with nitric acid, sulfuric acid, potassium permanganate, and potassium persulfate in polypropylene tubes in a water bath at 90 to 95 degrees Celsius $\left({ }^{\circ} \mathrm{C}\right)$. Before analysis, hydroxylamine hydrochloride was added to reduce excess permanganate, and the samples were brought to volume using distilleddeionized water.

In the CVAAS procedure for determination of $\mathrm{Hg}$, divalent mercury $\left(\mathrm{Hg}^{2+}\right)$ in aqueous samples (digests of tissue samples) is reduced to the elemental state $\left(\mathrm{Hg}^{0}\right)$ by a strong reducing agent (stannous chloride). Gaseous $\mathrm{Hg}^{0}$ enters the sweep gas and is introduced into an atomic absorption cell, where light produced by a $\mathrm{Hg}$ vapor lamp is absorbed by the free $\mathrm{Hg}$ atoms. Mercury concentration in the sample is determined by comparing light absorption of the sample with that of external calibration standards. The range limit of detection (LOD) for these analyses was 0.009 to 0.0613 micrograms per gram $(\mu \mathrm{g} / \mathrm{g})$, dry weight.

Procedures that require tissue samples to be freeze-dried to determine mercury concentrations result in removal of the original moisture in the sample. Therefore, it is necessary to determine tissue moisture content to provide an estimate of mercury concentration on a "live" or "wet weight" basis. Mercury concentration in tissue is regulated on wet weight basis (U.S. Food and Drug Administration, 1994; U.S. Environmental Protection Agency, 2001). Additionally, tissue moisture is a relative measure of the quality of the tissue sample.

Moisture content was determined by weight loss upon freeze drying and is expressed as weight percentage of the original wet sample. Depending on sample size, either the whole sample or a representative aliquot was frozen and then dried under vacuum until a constant weight was attained. Samples were prepared and dried using plastic materials to minimize potential contamination artifacts. 


\section{Quality Assurance and Quality Control}

As part of normal quality-assurance (QA) and quality-control (QC) procedures, a standard number of procedural blanks, laboratory duplicate samples, blind duplicate samples, spiked samples, and standard reference materials were analyzed for each set of samples. Three sets of fish samples from Trinity County were analyzed at the TERL during the period 20002002.

Procedural blanks were analyzed to quantify the amount of total mercury that may have been added inadvertently during sample processing. A total of 18 procedural blanks were analyzed in the three sample sets, with results ranging from 0.00001 to $0.0047 \mu \mathrm{g} / \mathrm{g}$ (dry weight); results were within acceptable limits for all the samples.

Laboratory replicate samples were analyzed to provide a measure of the precision of the methods used for analysis. After the sample was homogenized, two separate subsamples were taken and analyzed. Replicate analyses were evaluated in accordance with a two-tiered acceptance criterion as follows: if the sample concentration was within the range of 2 to 10 times the limit of detection (LOD), the variation in terms of a 95-percent confidence interval had to be within 20 percent of the original value to meet the criterion, or if the sample concentration was greater than 10 times the LOD, then the replicate sample had to be within 10 percent of the original value to fall within the 95-percent confidence interval.

Relative percentage difference (RPD) is a measure of variability or precision for replicate analyses, and is computed as 100 times the absolute value of the difference between two replicate analyses divided by the mean of the replicate analyses. Values of RPD for the 15 laboratory replicates analyzed in the three sample sets ranged from 0.24 to 9.17 percent, well within acceptable limits.

Spiked samples were analyzed to provide a measure of the accuracy of the methods used for analysis. After the sample was homogenized, two separate subsamples were taken: one was processed as a sample, and the other subsample had a known quantity of analyte added prior to analysis. Spike recoveries were considered acceptable if the average recovery was 85 to 115 percent of the spike concentration after subtraction of the sample concentration. For the 18 spiked samples in the three sample sets, the recovered percentage of the added spike total concentration ranged from 93.5 to 107 percent.

Standard reference material (SRM) was analyzed to provide an estimate of range in accuracy of the laboratory instrument used for the determination of total mercury concentration and to ensure that this method produced results that were comparable to those obtained by an independent organization. The SRM used by the TERL was dogfish (Squalus sp.) muscle (DORM-2), certified by the National Research Council Canada (NRCC) as having a certified reference value (CRV) of $4.64 \mu \mathrm{g} / \mathrm{g}$ mercury (dry). The percentage recovery for the 18 analyses of DORM-2 in the three sample sets ranged from 82.7 to 107 percent, which is within acceptable limits.

Blind replicate samples were submitted to the TERL as additional QA-QC check on laboratory procedures. A total of 39 blind replicate samples from the three sample sets were submitted to the TERL. Data for these analyses are listed in table 3. Most of the blind replicate samples showed little variation. The median value of RPD for the 39 replicates was 3.8 percent, and the mean value was 8.0 percent. Thirty-two of the 39 blind replicate pairs ( 82 percent) had RPD values less than 10 percent, and 36 of 39 pairs (92 percent) had RPD values less than 20 percent. These results are considered to be within acceptable limits of variability.

Table 3. Data from replicate analyses of fishes collected in Trinity County, California, 2000-2002

[Sample dissected from left fillet of fish; replicate sample dissected from right fillet of fish. Hwy, highway; Mt, Mount. Hg, mercury. HgT, total mercury. Sex: F, female; M, male; U, unknown. mm, millimeter; g, gram; \%, percent; $\mu \mathrm{g} / \mathrm{g}$, microgram per gram (equivalent to part per million)]

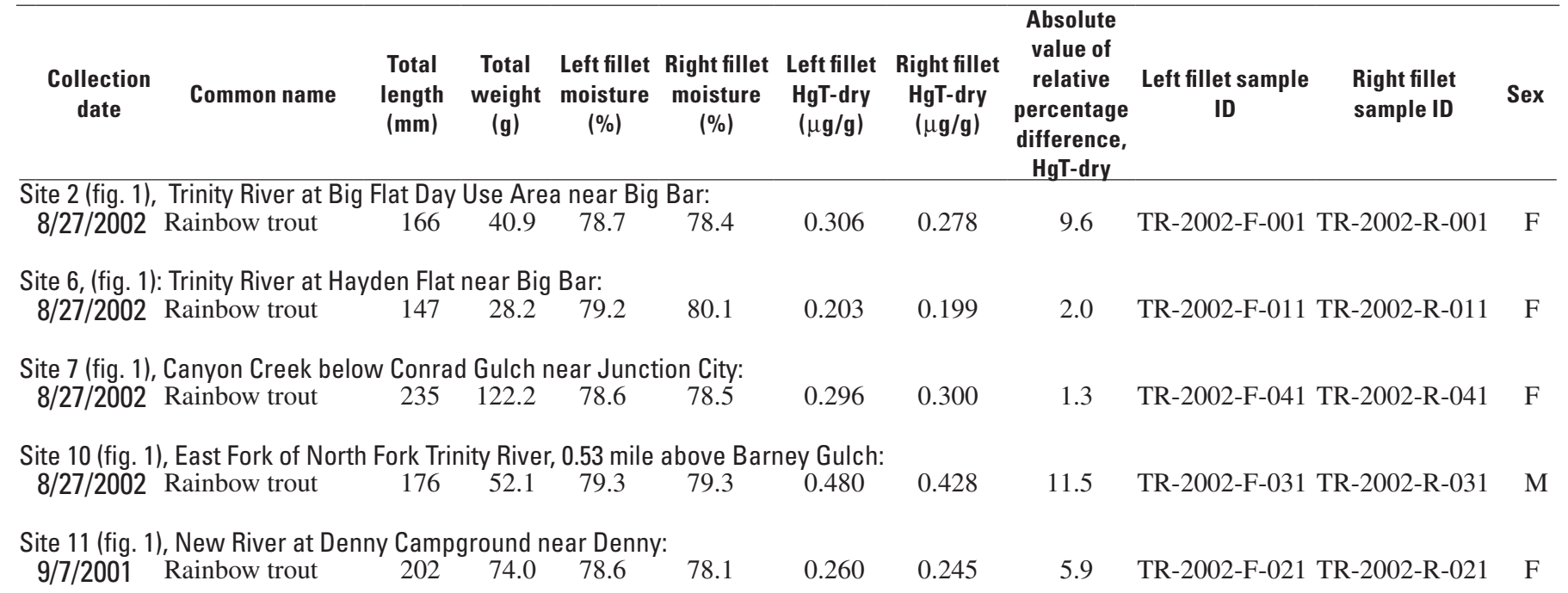


Table 3. Data from replicate analyses of fishes collected in Trinity County, California, 2000-2002 - Continued.

[Sample dissected from left fillet of fish; replicate sample dissected from right fillet of fish. Hwy, highway; Mt, Mount. Hg, mercury. HgT, total mercury. Sex: F, female; M, male; $\mathrm{U}$, unknown. $\mathrm{mm}$, millimeter; g, gram; \%, percent; $\mu \mathrm{g} / \mathrm{g}$, microgram per gram (equivalent to part per million)]

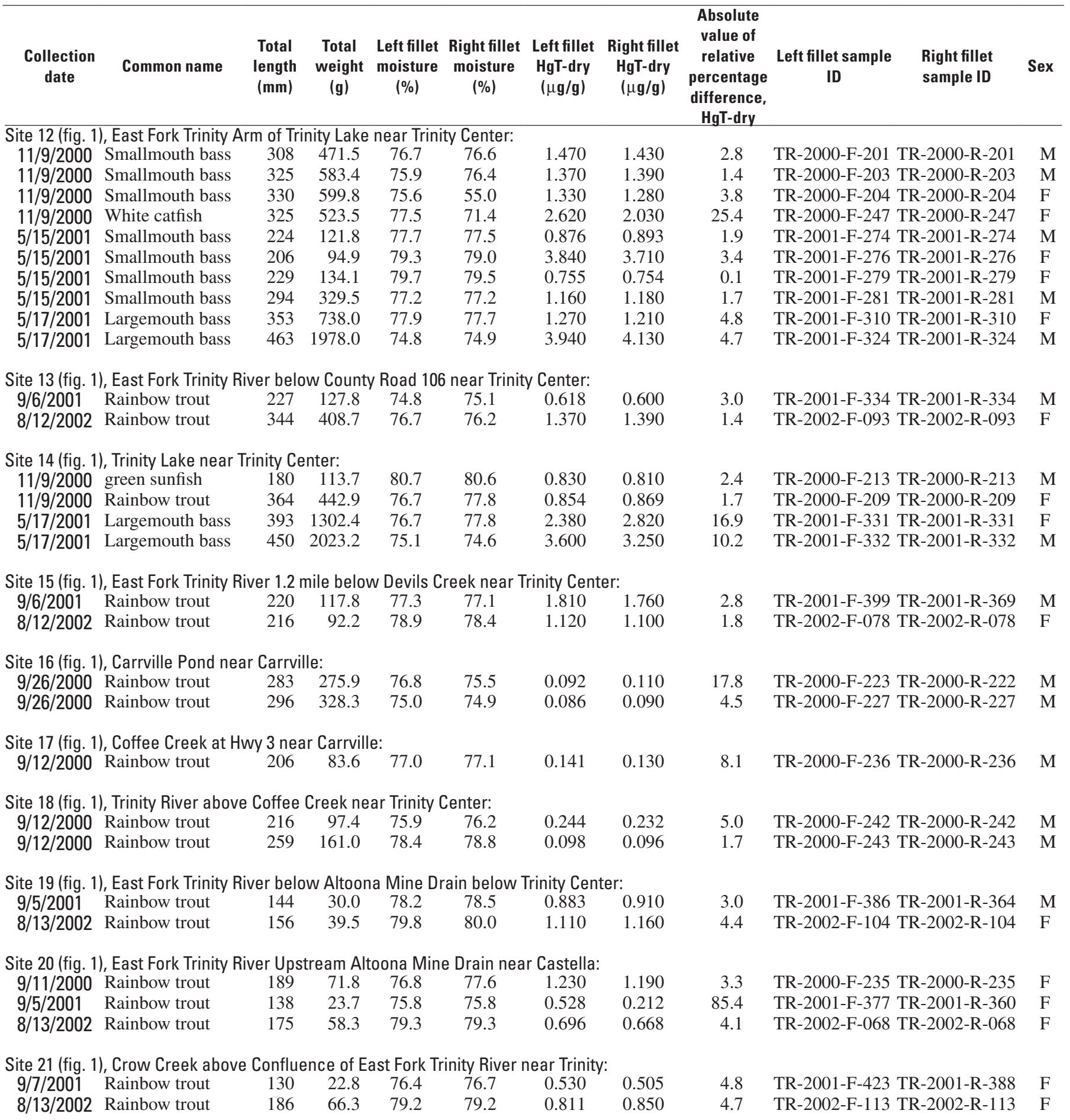


Table 3. Data from replicate analyses of fishes collected in Trinity County, California, 2000-2002 - Continued.

[Sample dissected from left fillet of fish; replicate sample dissected from right fillet of fish. Hwy, highway; Mt, Mount. Hg, mercury. HgT, total mercury. Sex: F, female; M, male; $\mathrm{U}$, unknown. mm, millimeter; g, gram; $\%$, percent; $\mu \mathrm{g} / \mathrm{g}$, microgram per gram (equivalent to part per million)]

\begin{tabular}{|c|c|c|c|c|c|c|c|c|c|c|c|}
\hline $\begin{array}{c}\text { Collection } \\
\text { date }\end{array}$ & Common name & $\begin{array}{c}\text { Total } \\
\text { length } \\
(\mathrm{mm})\end{array}$ & $\begin{array}{c}\text { Total } \\
\text { weight } \\
\text { (g) }\end{array}$ & $\begin{array}{c}\text { Left fillet } \\
\text { moisture } \\
(\%)\end{array}$ & $\begin{array}{c}\text { Right fillet } \\
\text { moisture } \\
(\%)\end{array}$ & $\begin{array}{c}\text { Left fillet } \\
\text { HgT-dry } \\
(\mu \mathrm{g} / \mathrm{g})\end{array}$ & $\begin{array}{c}\text { Right fillet } \\
\text { HgT-dry } \\
(\mu \mathrm{g} / \mathrm{g})\end{array}$ & $\begin{array}{c}\text { Absolute } \\
\text { value of } \\
\text { relative } \\
\text { percentage } \\
\text { difference, } \\
\text { HgT-dry } \\
\end{array}$ & $\begin{array}{l}\text { Left fillet sample } \\
\text { ID }\end{array}$ & $\begin{array}{l}\text { Right fillet } \\
\text { sample ID }\end{array}$ & Sex \\
\hline \multicolumn{12}{|c|}{ Site 22 (fig. 1), Tamarack Creek at East Fork Trinity River near Mt. Shasta: } \\
\hline 9/7/2001 & Rainbow trout & 178 & 50.2 & 81.3 & 81.4 & 0.604 & 0.600 & 0.7 & TR-2001-F-347 & TR-2001-R-340 & $\mathrm{M}$ \\
\hline $8 / 14 / 2002$ & Rainbow trout & 133 & 26.0 & 78.1 & 78.3 & 0.151 & 0.164 & 8.3 & TR-2002-F-054 & TR-2002-R-054 & $\mathrm{F}$ \\
\hline $9 / 7 / 2001$ & Brook trout & 230 & 144.7 & 77.2 & 80.5 & & 0.776 & 30.3 & TR-2001-F-371 & TR-2001-R-351 & $\mathrm{M}$ \\
\hline $9 / 7 / 2001$ & Rainbow trout & 169 & 34.3 & 81.3 & 83.1 & 1.170 & 1.210 & 3.4 & TR-2001-F-363 & TR-2001-R-350 & $\mathrm{U}$ \\
\hline
\end{tabular}

\section{Results}

A total of 368 fishes were collected and processed for the analysis of total mercury (table 2). Total mercury in 74 black bass (largemouth and smallmouth bass; Micropterus spp.) samples ranged from 0.046 to $1.225 \mu \mathrm{g} / \mathrm{g}$ (equivalent to parts per million or ppm) wet weight (ww). Mercury concentrations in 26 of the 34 black bass ( 76 percent) of legal catch size ( $\geq 305$ millimeters in length) were $\geq 0.3 \mathrm{ppm}$ (ww), the U.S. Environmental Protection Agency water-quality criterion for the protection of human health (U.S. Environmental Protection Agency, 2001). Mercury concentrations exceeded 1.0 ppm (ww), the Food and Drug Administration action level for commercial fish (U.S. Food and Drug Administration, 1994), in 3 of the 34 black bass ( 9 percent) of legal catch size. In contrast, only 3 of the 237 (about 1 percent) rainbow trout of all sizes sampled from stream, pond, and lake sites had $\mathrm{Hg}$ concentrations $\geq 0.3 \mathrm{ppm}$ (ww) (table 2).

In response to data generated by this study and other related investigations, the California Environmental Protection Agency's Office of Environmental Health Hazard Assessment (OEHHA) issued a draft fish-consumption advisory report that offered guidelines for human consumption of fish (Klasing and Brodberg, 2005). The final version of the OEHHA fishconsumption advisory (Klasing and Brodberg, in press) was approved by the State of California in July 2005 and is scheduled for publication in October 2005 (http://www.oehha.ca.gov/ fish/so_cal/TrinRiverF2.html)

\section{References}

Alpers, C.N., Hunerlach, M.P., May, J.T., and Hothem, R.L., 2005, Mercury contamination from historical gold mining in California: U.S. Geological Survey Fact Sheet 20053014, 6 p., accessed August 28, 2005, at http://water. usgs.gov/pubs/fs/2005/3014/.
Clark, W.B., 1963, Gold districts of California: California Department of Conservation, Division of Mines and Geology, Bulletin 193, 199 p., 1 plate (some revisions through 1969; seventh printing, 1998).

Klasing, S. and Brodberg, R., 2005, Draft health advisory: Fish consumption guidelines for Trinity Lake and selected water bodies in the Trinity River Watershed (Trinity County): California Environmental Protection Agency, Office of Environmental Health Hazard Assessment, April 2005, 45 p., accessed August 28, 2005, at http://www.oehha.ca.gov/fish/so_cal/pdf_zip/ TrinityLakeDraftAdvisory.pdf

May, J.T., Hothem, R.L., Alpers, C.N., and Law, M.A., 2000, Mercury bioaccumulation in fish in a region affected by historic gold mining: The South Yuba River, Deer Creek, and Bear River watersheds, California, 1999: U.S. Geological Survey Open-File Report 00-367, 30 p., available on the World Wide Web at http://ca.water.usgs. gov/archive/reports/ofr00367/

Swinney, C.M., 1950, The Altoona Quicksilver Mine, Trinity County, California: California Journal of Mines and Geology, v. 6, no. 3 .

U.S. Environmental Protection Agency, 1991, Methods for the determination of metals in environmental samples: Washington, D.C., EPA/600 4-91-010.

U.S. Environmental Protection Agency, 1995, Guidance for assessing chemical contaminant data for use in fish advisories, Volume 1. Fish Sampling and Analysis. 2nd edition: Washington, D.C., EPA823-R-95-007.

U.S. Environmental Protection Agency, 2001, Water quality criterion for the protection of human health: methyl mercury: Washington, D.C., EPA-823-R-01-001, accessed on July 1, 2005 at http://www.epa.gov/waterscience/criteria/ methylmercury/

U.S. Food and Drug Administration, 1994, Mercury in fish: Cause for concern?: FDA Consumer Magazine, v. 28, no 7, unnumbered pages, accessed on July 1, 2005, at http://www.fda.gov/fdac/reprints/mercury.html 

